\title{
Mesoscopic behavior from microscopic Markov dynamics and its application to calcium release channels
}

\author{
Nils Christian ${ }^{\mathrm{a}, \mathrm{b}}$, Alexander Skupin ${ }^{\mathrm{b}, \mathrm{c}}$, Silvia Moranted ${ }^{\mathrm{d}}$, Karl Jansen ${ }^{\mathrm{e}}$, Giancarlo Rossi ${ }^{\mathrm{d}}$, \\ Oliver Ebenhöh ${ }^{\mathrm{a}, \mathrm{f}, *}$ \\ ${ }^{a}$ University of Aberdeen, Department of Physics, Meston Walk, Aberdeen AB24 3UE, UK \\ ${ }^{b}$ University Luxembourg, Luxembourg Centre for Systems Biomedicine, 7, avenue des Hauts-Fourneaux, \\ L-4362 Esch-sur-Alzette, Luxembourg \\ ${ }^{c}$ Institute for Systems Biology, 401 Terry Ave N, Seattle, WA, 98109, USA \\ ${ }^{d}$ Università di Roma Tor Vergata and INFN, Sezione di Roma 2, Via della Ricerca Scientifica, I-00133 \\ Roma, Italy \\ ${ }^{e}$ NIC/DESY Zeuthen, Platanenallee 6, D-15738 Zeuthen, Germany \\ ${ }^{f}$ Helmholtz Centre Potsdam, GFZ German Research Centre for Geosciences, 14473 Potsdam, Germany
}

\begin{abstract}
A major challenge in biology is to understand how molecular processes determine phenotypic features. We address this fundamental problem in a class of model systems by developing a general mathematical framework that allows the calculation of mesoscopic properties from the knowledge of microscopic Markovian transition probabilities. We show how exact analytic formulae for the first and second moments of resident time distributions in mesostates can be derived from microscopic resident times and transition probabilities even for systems with a large number of microstates. We apply our formalism to models of the inositol trisphosphate receptor, which plays a key role in generating calcium signals triggering a wide variety of cellular responses. We demonstrate how experimentally accessible quantities, such as opening and closing times and the coefficient of variation of inter-spike intervals, and other, more elaborated, quantities can be analytically calculated from the underlying microscopic Markovian dynamics. A virtue of our approach is that we do not need to follow the detailed time evolution of the whole system, as we derive the relevant properties of its steady state without having to take into account the often extremely complicated transient features. We emphasize that our formulae fully agree with results obtained by stochastic simulations and approaches based on a full determination of the microscopic system's time evolution. We also illustrate how experiments can be devised to discriminate between alternative molecular models of the inositol trisphosphate receptor. The developed approach is applicable to any system described by a Markov process and, owing to the analytic nature of the resulting formulae, provides an easy way to characterize also rare events that are of particular importance to understand the intermittency properties of complex dynamic systems.
\end{abstract}

Keywords: calcium signaling, $\mathrm{IP}_{3}$ receptor, emergent properties of mesostates, Gillespie algorithm

${ }^{*}$ Corresponding author. Tel. +447580078809

Email addresses: nils.christian@uni.lu (Nils Christian), ebenhoeh@abdn.ac.uk (Oliver Ebenhöh) 


\section{Introduction}

A major problem in biology is how characteristic features of living systems can be explained as emergent properties from underlying, elementary physico-chemical processes (Schrödinger, 1944). The rapid technological advancements in the field of molecular biology allow observation of phenomena at many scales simultaneously. Thus, not surprisingly, in the last decades again a strong focus has been put on the occurrence of emerging properties in self-organizing systems, the understanding of which is essential for a more complete picture of life (Laughlin and Pines, 2000).

A fruitful approach to study complex systems, which include biological systems as prominent examples, is by scale separation, separating the dynamics on a microscopic scale from that on a mesoscopic or macroscopic scale at which the elementary, microscopic subunits are functionally organized (Mézard et al., 1987). The definition of what has to be considered as "microscopic" depends on the scale at which observations are made. For molecular interaction networks, for example, elementary subunits can be atoms or molecules, the configuration of which define the mesoscopic state of a cell. In population dynamics single cells or organisms should be considered as elementary units, the interplay of which gives rise to the mesoscopic dynamics on the population level. This scale separation allows decoupling the "fast" dynamics of the self-interacting elementary constituents from the "slower" evolution of the emergent mesoscopic degrees of freedom, driven or influenced by external "forces".

The notion of scale separation is very useful because it provides a conceptually appealing and mathematically consistent way to model the system at different complexity scales. At a lower scale level the number of relevant degrees of freedom is usually quite large and the network of their interactions is extremely complicated. Taking inspiration from the "central limit theorem", one can hope to be able to describe the system's underlying micro-dynamics by some sort of simple stochastic process, most often of the Markov type, that is intended to model the complicated network of individual molecular interactions. At a higher and more structured level, new collective degrees of freedom emerge from the more basic ones, that are in turn driven by external "forces", such as temperature, $\mathrm{pH}$, concentrations or gate potentials, eventually back-reacting on the microscopic dynamics. Of course many variations of this general scheme are possible and have been explored in the literature with applications to the description of channel activation (Shuai et al., 2007; Colquhoun and Hawkes, 1982), neuronal activity (Schwalger and Lindner, 2010), organelle interaction within the cell (Heinrich and Rapoport, 2005), homopolymer folding (La Penna et al., 2004), immunological response (Parisi, 1990) and many other processes.

In this paper we develop a general mathematical formalism able to describe the micro-tomeso transition step, showing how from the underlying microscopic Markovian dynamics it is possible to derive fairly simple analytic formulae characterizing and describing the emergence of higher level mesoscopic properties. The key feature of the present approach is that one can directly compute steady-state properties of mesostates, which we define as any ensemble of microstates, without the need of following the whole time-dependent transient dynamics of the system. Our analytic formulae are directly derived from the underlying Markovian dynamics by considering all possible paths of the system. Despite the straight-forward nature of our approach, we are not aware of any analogous method previously published. An approach that is based on following in detail the dynamics of micro- and mesostates (though 
the authors do not call them this way) has been developed in the seminal paper of Colquhoun and Hawkes (1982), which is based on analysing the system dynamics in terms of the Laplacetransform of the time evolution equations. The time evolution of microstates is guided by the standard "master equation", that is in turn ruled by the elementary Markovian transition probabilities between pairs of microstates, while the time evolution of mesostates is driven by non-Markovian equations resulting from summing over the microstates defining each one of the mesostates. Applications and the further development of this approach have been reviewed in Ball et al. (2000) and have been applied in a variant in Moenke et al. (2012). A notable difference of our method, compared to other approaches, is that it provides an efficient way to compute the first statistical moments of the observable mesoscopic process for an underlying arbitrarily complex microscopic dynamics, as it avoids inversion of the often extremely large transition probability matrices encoding the elementary underlying Markov processes, by breaking them down into smaller sub-matrices. In this way one is in position to more easily discriminate among different models by comparing experimentally measurable moment relations with their analytic expressions.

We illustrate our concepts and methods by studying the opening and closing dynamics of calcium driven channels of which the so-called inositol trisphosphate receptor $\left(\mathrm{IP}_{3}\right.$ receptor, or $\mathrm{IP}_{3} \mathrm{R}$ ) (Allbritton et al., 1992; Bezprozvanny and Ehrlich, 1994) is an important and intensively studied biological prototype. The $\mathrm{IP}_{3}$ pathway is a predominant release mechanism of calcium from intracellular stores and an important physiological second messenger pathway (Berridge et al., 1998). The release is induced by moderate increase of the cytosolic calcium concentrations, known as calcium induced calcium release (CICR), but inhibited by higher calcium concentrations (Bezprozvanny et al., 1991). The release is countered by an ATP-dependent transport of free calcium back into storage compounds by SERCA pumps (MacLennan et al., 1997). A spatially inhomogeneous distribution of calcium channels leads to a strong local coupling with the consequence that the stochastic behavior of single channels influences the whole-cell behavior (Skupin et al., 2008, 2010). Thus, the $\mathrm{IP}_{3}$ receptors form the microscopic basis for the generation of complex mesoscopic calcium signaling behavior (Berridge, 1997; Falcke and Malchow, 2003; Skupin and Falcke, 2009).

We select this particular example because a) it represents an important biological process required for the generation of calcium signals, b) the underlying microscopic dynamics can be described as a Markov process, c) mesostates and their statistical properties, such as opening times, inter opening event intervals (inter-spike intervals) and their corresponding coefficients of variation (CV), are important and experimentally measurable output quantities and d) more complex mesostates can be defined whose properties are highly illustrative for understanding how higher level properties emerge from the underlying microscopic structure. We first present in detail the theory of dwell times in mesoscopic states, which allows analytic calculations of the first and second moments of resident time distributions from the underlying Markovian dynamics of the microstates. We then apply our approach to several alternative models of the $\mathrm{IP}_{3}$ receptor dynamics and show how predicted moment relations can be used for model discrimination. 


\section{Mesostates: definition and transition probabilities}

We consider a general Markovian system with $s$ microstates. Let $k_{m n}$ denote the rate constant for the elementary transition from microstate $m$ to $n$ and $p_{m}$ the stationary probability of being in microstate $m$. Then the stationary probability rates, $v_{m n}$, are defined by

$$
v_{m n}=p_{m} k_{m n} .
$$

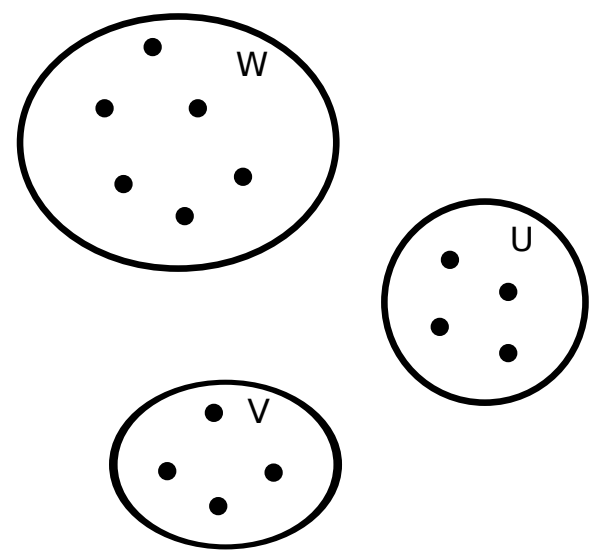

Figure 1: The configuration space of a Markov system. Small filled circles denote microstates. Sets of microstates enclosed by a curve correspond to mesostates.

Stationarity means that the probability of being in a particular microstate remains constant in time. This requires that the stationary probability rates for transitions to a microstate must be balanced by the rates for transitions from that state. This condition is expressed by the sum rule

$$
\sum_{m} v_{m n}=\sum_{l} v_{n l} .
$$

Using Eq. 1, the vector of stationary probabilities is obtained as a solution of the linear system

$$
\sum_{m} p_{m}\left(k_{m n}-\delta_{m n} \sum_{l} k_{m l}\right)=0 .
$$

The corresponding mean dwell (also called "residence") time in a microstate is given by

$$
\tau_{m}=\frac{p_{m}}{\sum_{n^{\prime}} v_{m n^{\prime}}}=\frac{1}{\sum_{n^{\prime}} k_{m n^{\prime}}},
$$

in terms of which the transition probability between microstates $m$ and $n$ can be expressed as

$$
\pi_{m n}=\frac{k_{m n}}{\sum_{n^{\prime}} k_{m n^{\prime}}}=\tau_{m} k_{m n} .
$$

We define mesostates as sets of microstates (see Fig. 1). If not stated otherwise, we assume that mesostates are finite and non-overlapping. From the elementary (microscopic) quantities 
defined above we will derive transition probabilities $(P)$ between mesostates and dwell times $(T)$ in mesostates, which are the essential properties of the process. These quantities depend on the history of the system and it is therefore crucial to take into account the possibility that certain transitions between micro and/or mesostates may have occurred before entering or are occurring after leaving a particular mesostate.

We use the convention that mesostates are denoted by upper case letters whereas microstates are denoted by lower case letters and are indicated as subscripts, as already used in Eqs. 1 5.

\subsection{Probabilities for mesostate transitions}

A transition between two mesostates $U$ and $V$ is defined as any series of microstate transitions that lead from one mesostate $(U)$ to the other mesostate $(V)$ without visiting a microstate not belonging to either $U$ or $V$. To characterize the mesoscopic observable dynamics, we have to calculate transition rates which are non-Markovian and therefore in general depend on the history of the system. The history dependence of the system is reflected by conditional probabilities that take the different microscopic transition paths into account.

Probability that next mesostate is $V$ when system is in $i \in U$. This quantity is the probability that for a system in a microstate $i$ of the mesostate $U$ the next mesostate transition will lead to $V$, and is computed as the sum of the probability of leaving $U$ directly from $i$ to $V$ plus that of leaving to $V$ after freely moving within $U$ (see Fig. 2 for an illustration). It can thus

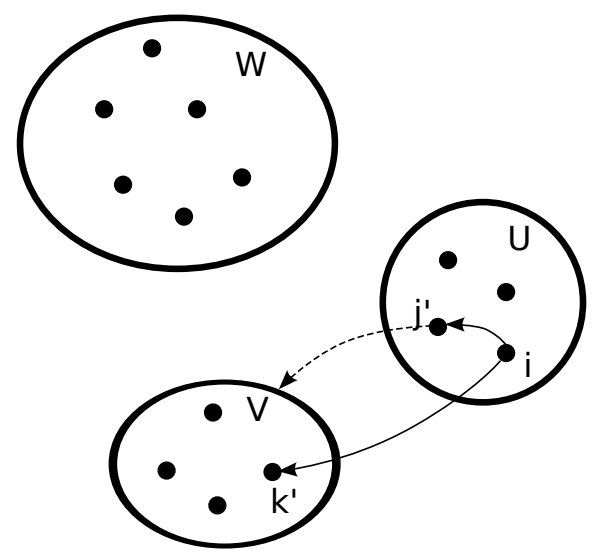

Figure 2: Different paths leading to a $U \rightarrow V$ mesostate transition starting from microstate $i \in U$. Two classes of possible events can occur: either the system visits another microstate $j^{\prime}$ in $U$, or the system leaves directly to $k^{\prime} \in V$

be calculated recursively by the formula

$$
P(U \rightarrow V \mid i \in U)=\sum_{k^{\prime} \in V} \pi_{i k^{\prime}}+\sum_{j^{\prime} \in U} \pi_{i j^{\prime}} P\left(U \rightarrow V \mid j^{\prime} \in U\right) \quad=\sum_{j^{\prime} \in U}(\mathbf{1}-\pi)_{i j^{\prime}}^{-1} \sum_{k^{\prime} \in V} \pi_{j^{\prime} k^{\prime}},
$$

where $\pi=\left(\pi_{i j}\right)$ is the matrix with coefficients $\pi_{i j}$. The recursive nature of Eq. 6 enables for an efficient calculation of the first statistical moments of the mesoscopic process generated by arbitrary complex microscopic dynamics. 
To introduce a notation that can be used for more complex transition chains we write Eq. 6 in the more transparent form

$$
\begin{aligned}
P(U \rightarrow V \mid i \in U) & =\sum_{k^{\prime} \in V} P\left(i \in U \rightarrow k^{\prime} \in V\right) \\
& +\sum_{j^{\prime} \in U} P\left(i \in U \rightarrow j^{\prime} \in U \wedge U \rightarrow V\right),
\end{aligned}
$$

where $P\left(i \in U \rightarrow j^{\prime} \in U \wedge U \rightarrow V\right)$ denotes the probability that the system will undergo a direct transition from $i \in U$ to $j^{\prime} \in U$ and that the next visited mesostate is $V$ (this leads to the second term of Eq. 6). Similarly, $P\left(i \in U \rightarrow k^{\prime} \in V\right)$ denotes the probability that the system will undergo a direct transition from $i \in U$ to $k^{\prime} \in V$, implying that the next visited mesostate is $V$ (and therefore equals $\pi_{i k^{\prime}}$ ).

Probability that first visited microstate in $V$ is $k$ when system is in $i \in U$ and the next mesostate is $V$. With the help of Eq. 6 we derive the probability that the next mesostate transition from microstate $i \in U$ arrives in microstate $k \in V$, under the condition that the next mesostate transition is $U \rightarrow V$. Similar to Eq. 6, we get a recursive formula

$$
\begin{aligned}
P(U \rightarrow k \in V \mid i \in U \wedge U \rightarrow V)= & P(i \in U \rightarrow k \in V \mid U \rightarrow V) \\
& +\sum_{j \in U} P(i \in U \rightarrow j \in U \mid U \rightarrow V) P(U \rightarrow k \in V \mid j \in U \wedge U \rightarrow V),
\end{aligned}
$$

where the first summand describes a direct transition to $V$, without visiting any other microstate within $U$, and can be calculated by normalizing the unconditional probability $\pi_{i k}$ with the probability that the next mesostate is $V$

$$
P(i \in U \rightarrow k \in V \mid U \rightarrow V)=\frac{\pi_{i k}}{P(U \rightarrow V \mid i \in U)}
$$

The second summand takes into account the alternative process of non-direct transitions in which the system visits another state $j \neq i$ within $U$ before leaving to $V$. With $M_{i j}$ defining the matrix elements (for a detailed derivation of the second summand see Supplementary Material S1.1)

$$
M_{i j}=P(i \in U \rightarrow j \in U \mid U \rightarrow V)=\left(1-\frac{\sum_{k^{\prime} \in V} \pi_{i k^{\prime}}}{P(U \rightarrow V \mid i \in U)}\right) \frac{\pi_{i j} P(U \rightarrow V \mid j \in U)}{\sum_{i^{\prime} \in U} \pi_{i i^{\prime}} P\left(U \rightarrow V \mid i^{\prime} \in U\right)},
$$

the solution of the recursive formula Eq. 7 reads

$$
P(U \rightarrow k \in V \mid i \in U \wedge U \rightarrow V)=\sum_{j \in U}(\mathbf{1}-\mathbf{M})_{i j}^{-1} \frac{\pi_{j k}}{P(U \rightarrow V \mid j \in U)} .
$$


Probability that mesostate transition from $W$ to $U$ arrives in microstate $i \in U$. To calculate the probability of arriving in $i \in U$, all stationary probability rates from any state in $W$ to $i \in U$ are summed up and normalized by all stationary probability rates from $W$ to any state $j^{\prime}$ in $U$, leading to

$$
P(W \rightarrow i \in U \mid W \rightarrow U)=\frac{\sum_{g \in W} p_{g} k_{g i}}{\sum_{g^{\prime} \in W} \sum_{j^{\prime} \in U} p_{g^{\prime}} k_{g^{\prime} j^{\prime}}} .
$$

In contrast to expressions 6 and 10 , this probability is conditional on a particular mesostate transition (i.e. not only on microstate properties). Therefore, it can only be determined under the assumption of stationarity and depends on the stationary probabilities.

\subsection{Transitions between mesostate subsets}

In some cases we might be interested in subsets of mesostates and transition between them. To derive statistical properties of such a subclass of mesostates, we need to know probabilities and dwell times of transition chains passing through mesostate $V$, where the first visited microstate in $V$ is from a subset $V^{\prime} \subset V$. We denote such a transition chain by

$$
\cdots \rightarrow V^{\prime}: V \rightarrow \ldots
$$

Determination of the probability of the transition chain $U \rightarrow V^{\prime}: V \rightarrow Z$ when currently in $i \in U$ involves summing only over the microstates of $V^{\prime}$

$$
P\left(U \rightarrow V^{\prime}: V \rightarrow Z \mid i \in U\right)=\sum_{k \in V^{\prime}} P\left(U \rightarrow k \in V^{\prime} \mid i \in U\right) P\left(V \rightarrow Z \mid k \in V^{\prime}\right) .
$$

The first term ensures that the first microstate in $V$ is from $V^{\prime}$ (because the sum only includes microstates from $V^{\prime}$ ), the second term implicitly allows to move within the whole mesostate $V$ before the system finally leaves to $Z$.

\section{Mesostate dwell times and raw moments}

From the knowledge of the rate constants, $k_{m n}$, for microstate transitions, we derive in this section analytic formulae for the average and variance of dwell times in mesostates. Because the dwell times are in general not Markovian variables, their values will depend on the particular microstate transition by which the mesostate was first entered. In general, the dwell times depend both on the microstate from which the previous mesostate was left and on the microstate through which the current mesostate was entered. We will denote dwell times in a mesostate by $T(U \mid \ldots)$.

The derivation of mesostate dwell times is not limited to a single mesostate, but can also be performed for consecutive mesostates. For example, the dwell time in the two consecutive mesostates $U$ and $V$ (i.e. the dwell time in $U$ plus the dwell time in the subsequent $V$ ) will be denoted by $T(U \rightarrow V \mid \ldots)$. 
Time spent in mesostate $U$ when system is in $i \in U$. The time spent in mesostate $U$ from the moment the system first arrived in $i \in U$ is given by the recursive equation (see Eq. 4)

$$
T(U \mid i \in U)=\tau_{i}+\sum_{j^{\prime} \in U} \pi_{i j^{\prime}} T\left(U \mid j^{\prime} \in U\right)
$$

which leads to

$$
T(U \mid i \in U)=\sum_{j^{\prime} \in U}(\mathbf{1}-\pi)_{i j^{\prime}}^{-1} \tau_{j^{\prime}}
$$

To calculate the second raw moments of dwell times, one has to distinguish between consecutive events and alternative events which occur with certain probabilities. The microscopic dwell times of two consecutive events are independent random variables. In general, the second raw moment of the sum of two independent random variables, $X_{a}$ and $X_{b}$, is given by $\left\langle\left(X_{a}+X_{b}\right)^{2}\right\rangle=\left\langle X_{a}^{2}\right\rangle+\left\langle X_{b}^{2}\right\rangle+2\left\langle X_{a}\right\rangle\left\langle X_{b}\right\rangle$. The second raw moment of the expected dwell time of two alternative processes is simply the mean of the individual second raw moments weighted with their probabilities. Thus, the second raw moment for the dwell time distribution in a mesostate (Eq. 13) is

$$
\begin{aligned}
T^{(2)}(U \mid i \in U)= & \left(1-\sum_{j^{\prime} \in U} \pi_{i j^{\prime}}\right) \tau_{i}^{(2)} \\
& +\sum_{j^{\prime} \in U} \pi_{i j^{\prime}}\left(\tau_{i}^{(2)}+T^{(2)}\left(U \mid j^{\prime} \in U\right)+2 \tau_{i} T\left(U \mid j^{\prime} \in U\right)\right) \\
= & \tau_{i}^{(2)}+\sum_{j^{\prime} \in U} \pi_{i j^{\prime}}\left(T^{(2)}\left(U \mid j^{\prime} \in U\right)+2 \tau_{i} T\left(U \mid j^{\prime} \in U\right)\right),
\end{aligned}
$$

where $\tau_{i}^{(2)}$ denotes the second raw moment of the microscopic dwell time $\tau_{i}$. The above formula simplifies if we assume that the microscopic dwell times have an exponential probability distribution, so that $\tau_{i}^{(2)}=2 \tau_{i}^{2}$. In this case

$$
\begin{aligned}
T^{(2)}(U \mid i \in U) & =2 \tau_{i}^{2}+\sum_{j^{\prime} \in U} \pi_{i j^{\prime}}\left(T^{(2)}\left(U \mid j^{\prime} \in U\right)+2 \tau_{i} T\left(U \mid j^{\prime} \in U\right)\right) \\
& =2 \tau_{i} \underbrace{\left(\tau_{i}+\sum_{j^{\prime} \in U} \pi_{i j^{\prime}} T\left(U \mid j^{\prime} \in U\right)\right)}_{T(U \mid i \in U)}+\sum_{j^{\prime} \in U} \pi_{i j^{\prime}} T^{(2)}\left(U \mid j^{\prime} \in U\right),
\end{aligned}
$$

leading to

$$
T^{(2)}(U \mid i \in U)=2 \sum_{j^{\prime} \in U}(\mathbf{1}-\pi)_{i j^{\prime}}^{-1} \tau_{j^{\prime}} T\left(U \mid j^{\prime} \in U\right) .
$$

Time spent in mesostate $U$ when system is in $i \in U$ and the next visited mesostate $i s ~ V$. The computation exactly parallels the line of arguments developed in the derivation of Eq. 10 and leads to the equation

$$
T(U \mid i \in U \wedge U \rightarrow V)=\tau_{i}+\sum_{j \in U} \underbrace{P(i \in U \rightarrow j \in U \mid U \rightarrow V)}_{M_{i j}} T(U \mid j \in U \wedge U \rightarrow V),
$$


which has the solution

$$
T(U \mid i \in U \wedge U \rightarrow V)=\sum_{j \in U}(\mathbf{1}-\mathbf{M})_{i j}^{-1} \tau_{j}
$$

Similar to the derivation of Eq. 16 one finds for the second raw moment

$$
\begin{aligned}
& T^{(2)}(U \mid i \in U \wedge U \rightarrow V)=\frac{\sum_{k^{\prime} \in V} \pi_{i k^{\prime}}}{P(U \rightarrow V \mid i \in U)} \tau_{i}^{(2)} \\
& +\sum_{j \in U} \underbrace{P(i \in U \rightarrow j \in U \mid U \rightarrow V)}_{M_{i j}} \times\left(\tau_{i}^{(2)}+T^{(2)}(U \mid j \in U \wedge U \rightarrow V)+2 \tau_{i} T(U \mid j \in U \wedge U \rightarrow V)\right) .
\end{aligned}
$$

For exponentially distributed $\tau_{i}$, one gets

$$
\begin{aligned}
T^{(2)}(U \mid i \in U \wedge U \rightarrow V) & =2 \tau_{i}^{2}+\sum_{j \in U} M_{i j}\left(T^{(2)}(U \mid j \in U \wedge U \rightarrow V)+2 \tau_{i} T(U \mid j \in U \wedge U \rightarrow V)\right) \\
& =2 \tau_{i} \underbrace{\left(\tau_{i}+\sum_{j \in U} M_{i j} T(U \mid j \in U \wedge U \rightarrow V)\right)}_{T(U \mid i \in U \wedge U \rightarrow V)}+\sum_{j \in U} M_{i j} T^{(2)}(U \mid j \in U \wedge U \rightarrow V)
\end{aligned}
$$

finally leading to

$$
T^{(2)}(U \mid i \in U \wedge U \rightarrow V)=2 \sum_{j \in U}(\mathbf{1}-\mathbf{M})_{i j}^{-1} \tau_{j} T(U \mid j \in U \wedge U \rightarrow V) .
$$

\section{Application to models of the $\mathrm{IP}_{3} \mathrm{R}$ channel}

$\mathrm{IP}_{3} \mathrm{R}$ channels are the elementary building blocks of calcium $\left(\mathrm{Ca}^{2+}\right)$ signals (Berridge, 1997) and thus play a crucial role as physiological signal mediators. Despite a lot of effort devoted to finding a mechanistic interpretation of their properties (De Young and Keizer, 1992 Gin et al., 2009), our understanding is still rather poor. We apply the general formalism developed in the previous sections to three interesting $\mathrm{IP}_{3} \mathrm{R}$ models. We show how, from the basic processes defined by these models, important mesoscopic physico-chemical properties of the system can be obtained by our approach. These include first and second raw moments of opening and closing time distributions and coherence (quantified by the coefficient of variation) of inter-spike intervals. Comparison of theoretical results for such quantities with experiments (Dellis et al., 2006; Rahman et al., 2009) may allow to identify the correct model of receptor structure and dynamics. Similar channel properties have been subjected to theoretical investigations (Higgins et al., 2009) aimed at a deeper understanding of cell physiology.

Model descriptions. In the popular and comprehensive De Young-Keizer model (De Young and Keizer, 1992) $\mathrm{IP}_{3}$ Rs are assumed to consist of four identical (independent) subunits, each endowed with three kinds of binding sites: a sensitizing $\mathrm{IP}_{3}$ binding site, an activating and a deactivating $\mathrm{Ca}^{2+}$ binding site. Since every site is either occupied or unoccupied, every 
subunit can reside in one of 8 states. We therefore term this model the 8 -state model. The $\mathrm{IP}_{3} \mathrm{R}$ releases $\mathrm{Ca}^{2+}$ from the endoplasmic reticulum if at least three of the four subunits are active, i.e. if the $\mathrm{IP}_{3}$ and the activating $\mathrm{Ca}^{2+}$ binding sites are occupied and the deactivating $\mathrm{Ca}^{2+}$ binding site is unoccupied. Due to the release of $\mathrm{Ca}^{2+}$ its concentration in the cytosol increases and can cause the deactivation of the subunits by binding back to the deactivating site.

Shuai et al. (2007) have argued that with such a simple model some important experimentally established results cannot be explained and therefore proposed a model with a further activating state for each subunit, which we refer to as the 9-state model. A key difference with the 8 -state model is that in the 9 -state model the deactivation of a single subunit is independent on the ligand concentrations and allows for a fast modulation of the open state. The main features of the two alternative models are summarized in Fig. 3.

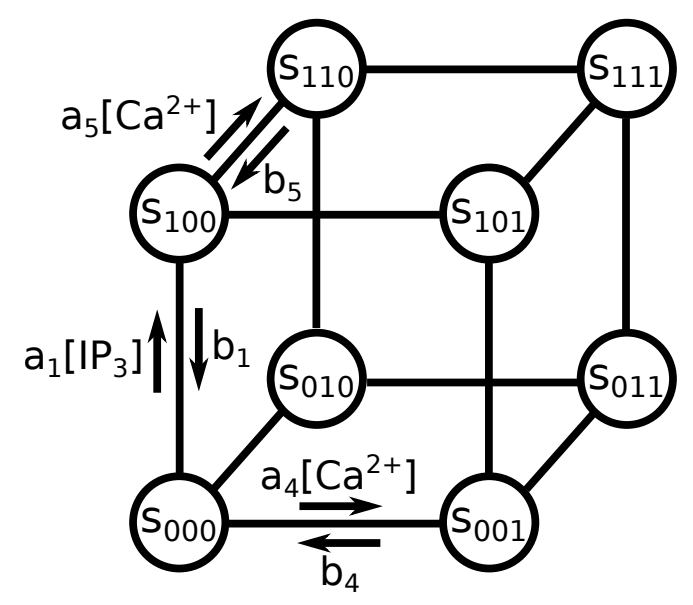

(a) 8-state model

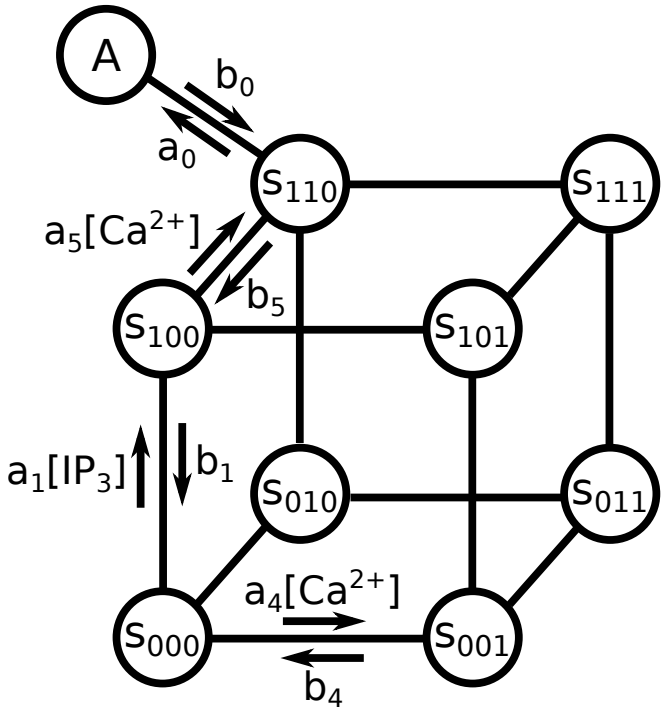

(b) 9-state model

Figure 3: Schematic representation of microstate transitions of a single $\mathrm{IP}_{3} \mathrm{R}$ subunits in the (a) 8-state and (b) 9-state model. Horizontal transitions denote binding and release of $\mathrm{Ca}^{2+}$, vertical transitions denote binding and release of $\mathrm{IP}_{3}$. The three binary digits denote the occupation state of the $\mathrm{IP}_{3}$, activating and deactivating $\mathrm{Ca}^{2+}$ binding site. In the 8-state model a subunit is active when it is in the state 110. In the 9-state model a subunit is active when from state 110 it undergoes a transition to the active state, A.

To calculate mesoscopic properties of the models we are going to exploit the stochastic analysis of probabilities and dwell times developed in the previous sections. To this end one has to first provide the rate constants $k_{i j}$ between each pair of microstates characterizing the model. The microstates are denoted by three binary digits corresponding to the occupation of the $\mathrm{IP}_{3}$, activating and deactivating $\mathrm{Ca}^{2+}$ binding site while A denotes the activated state. For a single subunit in the 9 -state model, the matrix $k$ reads (see Shuai et al. (2007)) 


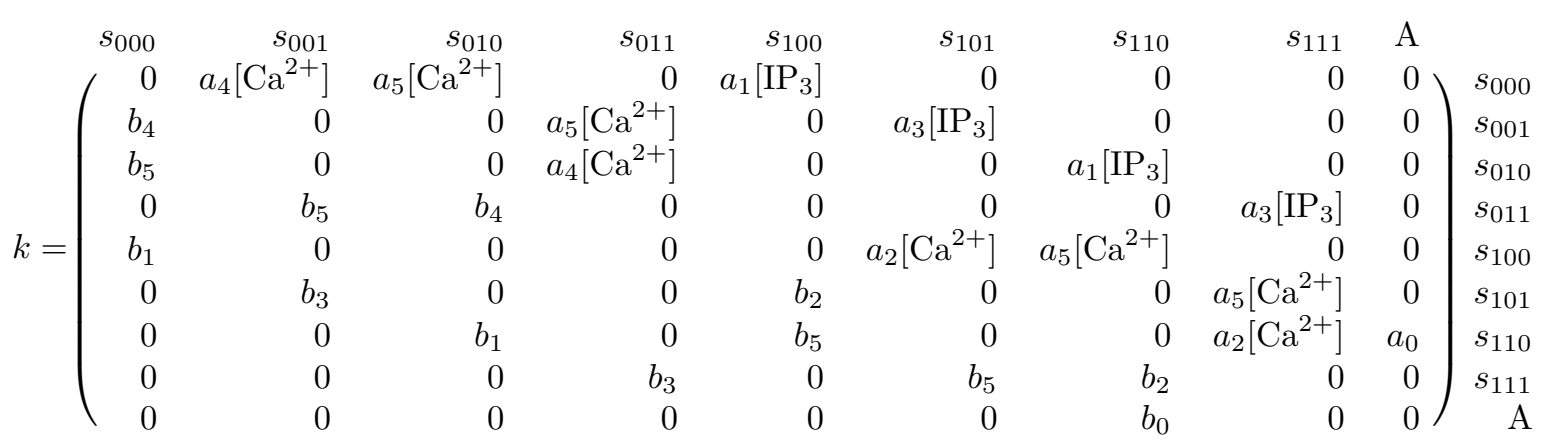

Naturally, in the absence of external fluxes, detailed-balance has to be obeyed by the elements of the matrix defined in Eq. 20, so that certain restrictions hold for the parameters $a_{i}$ and $b_{i}$ (for details see Shuai et al. (2007)). A channel with four independent subunits can assume $9^{4}$ different microstates. Considering symmetries (i.e. assuming that the subunits are indistinguishable), the transitions for the full four-subunit model can be described by a $495 \times 495$ matrix. The single-subunit matrix for the 8-state model looks similar to the 9-state model, with the row/column related to the active state A removed, as a subunit is considered already active if it is in state 110 . The $8^{4}$ different microstates of the four-subunit model can be described by 330 microstates when symmetries are taken into account.

From a molecular perspective, the fast modulation of the channel opening may be more realistically considered as a whole molecule or protein phenomenon. Once the channel is in an "excitable" state with at least 3 active subunits, the protein may exhibit emergent dynamics leading to the final opening of the channel. Thus, we discuss as a third model a modification of the 8-state model which also displays a ligand-independent subunit deactivation. The difference with the models discussed above is, that instead of each subunit individually undergoing a conformational change, we assume that a channel opens by a joint conformational change of the four subunits, locking the ligands until the channel closes again. Therefore we refer to this model as the global-activation model.

To describe this global conformation change, we have to introduce new microstates reflecting open channels. In analogy to the 8-state model, we assume that conformation change is only possible if at least 3 subunits are in state 110. Channel opening and closing are 
described by the following additional microstate transitions

$$
\begin{aligned}
4 s_{110} \stackrel{a_{\mathcal{O}}}{\rightarrow} \mathcal{O}_{4} & \mathcal{O}_{4} \stackrel{b_{\mathcal{O}}}{\longrightarrow} 4 s_{110} \\
3 s_{110}+s_{000} \stackrel{a_{\mathcal{O}}}{\longrightarrow} \mathcal{O}_{3}^{s_{000}} & \mathcal{O}_{3}^{s_{000}} \stackrel{b_{\mathcal{O}}}{\longrightarrow} 3 s_{110}+s_{000} \\
3 s_{110}+s_{001} \stackrel{a_{\mathcal{O}}}{\longrightarrow} \mathcal{O}_{3}^{s_{001}} & \mathcal{O}_{3}^{s_{001}} \stackrel{b_{\mathcal{O}}}{\longrightarrow} 3 s_{110}+s_{001} \\
3 s_{110}+s_{010} \stackrel{a_{\mathcal{O}}}{\longrightarrow} \mathcal{O}_{3}^{s_{010}} & \mathcal{O}_{3}^{s_{010}} \stackrel{b_{\mathcal{O}}}{\longrightarrow} 3 s_{110}+s_{010} \\
3 s_{110}+s_{011} \stackrel{a_{\mathcal{O}}}{\longrightarrow} \mathcal{O}_{3}^{s_{011}} & \mathcal{O}_{3}^{s_{011}} \stackrel{b_{\mathcal{O}}}{\longrightarrow} 3 s_{110}+s_{011} \\
3 s_{110}+s_{100} \stackrel{a_{\mathcal{O}}}{\longrightarrow} \mathcal{O}_{3}^{s_{100}} & \mathcal{O}_{3}^{s_{100}} \stackrel{b_{\mathcal{O}}}{\longrightarrow} 3 s_{110}+s_{100} \\
3 s_{110}+s_{101} \stackrel{a_{\mathcal{O}}}{\longrightarrow} \mathcal{O}_{3}^{s_{101}} & \mathcal{O}_{3}^{s_{101}} \stackrel{b_{\mathcal{O}}}{\longrightarrow} 3 s_{110}+s_{101} \\
3 s_{110}+s_{111} \stackrel{a_{\mathcal{O}}}{\longrightarrow} \mathcal{O}_{3}^{s_{111}} & \mathcal{O}_{3}^{s_{111}} \stackrel{b_{\mathcal{O}}}{\longrightarrow} 3 s_{110}+s_{111} .
\end{aligned}
$$

Here, the symbol $\mathcal{O}_{3}^{s_{i j k}}$ denotes the open state with 3 subunits locked in the occupation state $s_{110}$ and the remaining subunit locked in the occupation state $s_{i j k}$. The symbol $\mathcal{O}_{4}$ denotes the open state with all 4 subunits locked in the $s_{110}$ microstate. As in the 9-state model we assume that the rate constants for activation $\left(a_{\mathcal{O}}\right)$ and deactivation $\left(b_{\mathcal{O}}\right)$ do not depend on the $\mathrm{Ca}^{2+}$ and $\mathrm{IP}_{3}$ concentrations. In total, this model has $8^{4}+8 \cdot 4+1$ microstates which can be reduced to 338 by taking symmetries into account.

Derivation of channel properties. In the 8- and 9-state models, the channel is open if at least three subunits are active. In the following we derive channel opening and closing properties in the general case where the number of channel subunits is $N$ and the channel is open when at least $K_{t h} \leq N$ of them are active. To this end it is convenient to define the mesostates (with the integers fulfilling $0<M<L \leq N$ )

$$
\begin{aligned}
& C \quad \text { set of microstates corresponding to a closed channel } \\
& \text { (between } 0 \text { and } K_{t h}-1 \text { subunits are active) } \\
& O \quad \text { set of microstates corresponding to an open channel } \\
& \text { ( } K_{t h} \text { or more subunits are active) } \\
& A_{L} \quad \text { set of microstates with } L \text { active subunits } \\
& A_{[M, L]} \text { set of microstates with } M \text { to } L \text { active subunits }
\end{aligned}
$$

We further introduce the notation $\cdots \rightarrow A_{L}: A_{[M, L]} \rightarrow \ldots$ to denote the fact that in a transition chain passing through $A_{[M, L]}$ the first visited microstate in that mesostate belongs to $A_{L}$ (see Sect. 2.2.).

Opening and closing probabilities, mean opening time. The basic probabilities for the channel being in an open or closed state are simply

$$
P(O)=\sum_{i \in O} p_{i} \quad P(C)=\sum_{i \in C} p_{i} .
$$

For the mean opening and closing time we have

$$
\mathcal{T}(O)=T(O \mid C \rightarrow O \rightarrow C),
$$




$$
\mathcal{T}(C)=T(C \mid O \rightarrow C \rightarrow O) .
$$

Time spent in an $A_{L}$-related opening event. An $A_{L}$-related opening event is defined by the fact that a maximum of $L$ active subunits with $L \in\left[K_{t h}, N\right]$ is reached during the time the channel is open. Although the dwell time and the corresponding probability are not directly measurable, their knowledge is nevertheless interesting because understanding how long a channel remains open in an $A_{L}$-related opening event and how probable such an event is may give useful insight on the extent the internal structure of a channel influences its global characteristic opening behavior. Such quantities are highly non-trivial but with our present approach, these and even more complicated quantities can be derived systematically. The probabilities and dwell times required here are similar or closely follow the derivation described in the general section introducing the mesostate subsets.

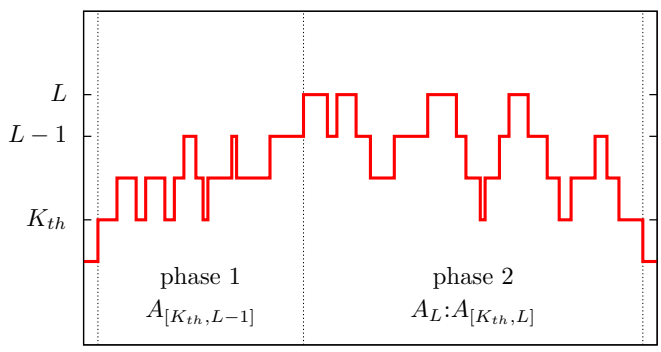

Figure 4: The time spent in an $A_{L}$-related opening event can be divided into two parts. During phase 1 a maximum of $L-1$ subunits are active. Phase 2 begins when for the first time $L$ subunits are active and ends when the channel closes.

First the dwell time in the mesostate $A_{\left[K_{t h}, L-1\right]}$ has to be calculated under the condition that from this mesostate only states with a maximal number of $L$ active subunits are visited before the system reaches the closed state $C$ (phase 1 in Fig. 4). For this we need the probability of the transition chain $A_{\left[K_{t h}, L-1\right]} \rightarrow A_{L}: A_{\left[K_{t h}, L\right]} \rightarrow C$ when currently in $i \in$ $A_{\left[K_{t h}, L-1\right]}$, which is (see Eq. 12)

$$
\begin{aligned}
& P\left(A_{\left[K_{t h}, L-1\right]} \rightarrow A_{L}: A_{\left[K_{t h}, L\right]} \rightarrow C \mid i \in A_{\left[K_{t h}, L-1\right]}\right)= \\
& \quad \sum_{l \in A_{L}} P\left(A_{\left[K_{t h}, L-1\right]} \rightarrow l \in A_{L} \mid i \in A_{\left[K_{t h}, L-1\right]}\right) P\left(A_{\left[K_{t h}, L\right]} \rightarrow C \mid l \in A_{\left[K_{t h}, L\right]}\right) .
\end{aligned}
$$

Using this expression, the probability that the first microstate in $A_{\left[K_{t h}, L-1\right]}$ is $i$, given the transition chain $C \rightarrow A_{\left[K_{t h}, L-1\right]} \rightarrow A_{L}: A_{\left[K_{t h}, L\right]} \rightarrow C$, can be written in the form (for details see Eq. S1.18 in the Supplementary Material S1)

$$
P\left(C \rightarrow i \in A_{\left[K_{t h}, L-1\right]} \mid C \rightarrow A_{\left[K_{t h}, L-1\right]} \rightarrow A_{L}: A_{\left[K_{t h}, L\right]} \rightarrow C\right)=\frac{P_{i}^{\text {phase } 1}}{\sum_{i^{\prime} \in A_{\left[K_{t h}, L-1\right]}} P_{i^{\prime}}^{\text {phase } 1}},
$$

where

$$
\begin{aligned}
P_{i}^{\text {phase 1 }}= & P\left(C \rightarrow i \in A_{\left[K_{t h}, L-1\right]} \mid C \rightarrow A_{\left[K_{t h}, L-1\right]}\right) \\
& \times P\left(A_{\left[K_{t h}, L-1\right]} \rightarrow A_{L}: A_{\left[K_{t h}, L\right]} \rightarrow C \mid i \in A_{\left[K_{t h}, L-1\right]}\right) .
\end{aligned}
$$


The time spent in phase 1 is therefore (see Eq. S1.17 in the Supplementary Material S1)

$$
\begin{aligned}
& T\left(A_{\left[K_{t h}, L-1\right]} \mid C \rightarrow A_{\left[K_{t h}, L-1\right]} \rightarrow A_{L}: A_{\left[K_{t h}, L\right]} \rightarrow C\right)= \\
& \sum_{i \in A_{\left[K_{t h}, L-1\right]}} P\left(C \rightarrow i \in A_{\left[K_{t h}, L-1\right]} \mid C \rightarrow A_{\left[K_{t h}, L-1\right]} \rightarrow A_{L}: A_{\left[K_{t h}, L\right]} \rightarrow C\right) \\
& \times T\left(A_{\left[K_{t h}, L-1\right]} \mid i \in A_{\left[K_{t h}, L-1\right]} \wedge A_{\left[K_{t h}, L-1\right]} \rightarrow A_{L}: A_{\left[K_{t h}, L\right]} \rightarrow C\right) .
\end{aligned}
$$

For computing the dwell time in phase 2 the conditional probability to arrive in microstate $l \in A_{L} \subset A_{\left[K_{t h}, L\right]}$, knowing that the next mesostate is closed (i.e. $C$ ), is needed. In analogy with Eq. 26 this probability can be derived to be

$$
P\left(A_{\left[K_{t h}, L-1\right]} \rightarrow l \in A_{L} \mid C \rightarrow A_{\left[K_{t h}, L-1\right]} \rightarrow A_{L}: A_{\left[K_{t h}, L\right]} \rightarrow C\right)=\frac{P_{l}^{\text {phase } 2}}{\sum_{l^{\prime} \in A_{L}} P_{l^{\prime}}^{\text {phase } 2}},
$$

with

$$
P_{l}^{\text {phase } 2}=P\left(A_{\left[K_{t h}, L-1\right]} \rightarrow l \in A_{L} \mid C \rightarrow A_{\left[K_{t h}, L-1\right]} \rightarrow A_{L}\right) P\left(A_{\left[K_{t h}, L\right]} \rightarrow C \mid l \in A_{\left[K_{t h}, L\right]}\right) .
$$

The time spent in phase 2 is therefore

$$
\begin{gathered}
T\left(A_{\left[K_{t h}, L\right]} \mid C \rightarrow A_{\left[K_{t h}, L-1\right]} \rightarrow A_{L}: A_{\left[K_{t h}, L\right]} \rightarrow C\right)= \\
\sum_{l \in A_{L}} P\left(A_{\left[K_{t h}, L-1\right]} \rightarrow l \in A_{L} \mid C \rightarrow A_{\left[K_{t h}, L-1\right]} \rightarrow A_{L}: A_{\left[K_{t h}, L\right]} \rightarrow C\right) \\
\times T\left(A_{\left[K_{t h}, L\right]} \mid l \in A_{\left[K_{t h}, L\right]} \wedge A_{\left[K_{t h}, L\right]} \rightarrow C\right) .
\end{gathered}
$$

Altogether, summing Eqs. 27 and 29, the mean time spent in an $A_{L}$-related opening event is

$$
\begin{aligned}
\mathcal{T}\left(A_{L}\right)= & T\left(A_{\left[K_{t h}, L-1\right]} \mid C \rightarrow A_{\left[K_{t h}, L-1\right]} \rightarrow A_{L}: A_{\left[K_{t h}, L\right]} \rightarrow C\right) \\
& +T\left(A_{\left[K_{t h}, L\right]} \mid C \rightarrow A_{\left[K_{t h}, L-1\right]} \rightarrow A_{L}: A_{\left[K_{t h}, L\right]} \rightarrow C\right) .
\end{aligned}
$$

Probability of an $A_{L}$-related opening event. We now seek for an expression for the probability of an $A_{L}$-related opening event, $\mathcal{P}\left(A_{L}\right)$, i.e. for the fraction of the opening events with a maximum of $L$ active subunits. To this end, we first have to compute the auxiliary probability of leaving $A_{\left[K_{t h}, L\right]}$ to the closed state (i.e. without visiting $A_{L+1}$ ), under the condition that we reached $A_{\left[K_{t h}, L\right]}$ from the closed state. For this auxiliary probability one finds

$$
\sum_{l^{\prime} \in A_{L}} P\left(A_{\left[K_{t h}, L-1\right]} \rightarrow l^{\prime} \in A_{L} \mid C \rightarrow A_{\left[K_{t h}, L-1\right]} \rightarrow A_{L}\right) P\left(A_{\left[K_{t h}, L\right]} \rightarrow C \mid l^{\prime} \in A_{\left[K_{t h}, L\right]}\right) .
$$

To obtain $\mathcal{P}\left(A_{L}\right)$ this has to be multiplied by the probability of arriving in $L$, finally leading to

$$
\begin{aligned}
\mathcal{P}\left(A_{L}\right)= & P\left(A_{\left[K_{t h}, L-1\right]} \rightarrow A_{L} \mid C \rightarrow A_{\left[K_{t h}, L-1\right]}\right) \\
& \times \sum_{l^{\prime} \in A_{L}} P\left(A_{\left[K_{t h}, L-1\right]} \rightarrow l^{\prime} \in A_{L} \mid C \rightarrow A_{\left[K_{t h}, L-1\right]} \rightarrow A_{L}\right) P\left(A_{\left[K_{t h}, L\right]} \rightarrow C \mid l^{\prime} \in A_{\left[K_{t h}, L\right]}\right) .
\end{aligned}
$$


Inter-spike interval. An important measurable quantity is the so-called inter-spike interval, $\mathcal{T}(I)$, i.e. the interval between two opening events. It can be calculated by the weighted average over all the dwell times in $O$ leading to a specific microstate in $C$, to which one has to add the subsequent dwell time in $C$. This leads to the expression

$$
\begin{aligned}
& T(O \rightarrow C \mid i \in O)= \\
& \sum_{j \in C}(P(O \rightarrow j \in C \mid i \in O \wedge O \rightarrow C)[T(O \mid i \in O \wedge O \rightarrow j \in C)+T(C \mid j \in C \wedge C \rightarrow O)]) .
\end{aligned}
$$

The associated second raw moment is consequently

$$
\begin{aligned}
& T^{(2)}(O \rightarrow C \mid i \in O)= \\
& \sum_{j \in C}\left(P ( O \rightarrow j \in C | i \in O \wedge O \rightarrow C ) \left[T^{(2)}(O \mid i \in O \wedge O \rightarrow j \in C)+T^{(2)}(C \mid j \in C \wedge C \rightarrow O)\right.\right. \\
& +2 T(O \mid i \in O \wedge O \rightarrow j \in C) T(C \mid j \in C \wedge C \rightarrow O)]) .
\end{aligned}
$$

To get quantities that are independent of the microstate $i$, Eqs. 33 and 34 have to be weighted with the probability of arriving in the microstate $i \in O$. One gets in this way for the interspike interval

$$
\mathcal{T}(I)=\sum_{i \in O} P(C \rightarrow i \in O \mid C \rightarrow O) T(O \rightarrow C \mid i \in O),
$$

and for its second raw moment

$$
\mathcal{T}^{(2)}(I)=\sum_{i \in O} P(C \rightarrow i \in O \mid C \rightarrow O) T^{(2)}(O \rightarrow C \mid i \in O) .
$$

From Eqs. 33 and 34 one can construct the coefficient of variation

$$
\mathrm{CV}(\mathcal{T}(I))=\frac{\left(\mathcal{T}^{(2)}(I)-(\mathcal{T}(I))^{2}\right)^{1 / 2}}{\mathcal{T}(I)}
$$

\section{Mesoscopic properties of the $\mathrm{IP}_{3} \mathrm{R}$ models}

In this section, using the analytical formulae derived in previous sections, we show results for the behavior of some of the observables we have defined before as function of the $\mathrm{Ca}^{2+}$ and/or $\mathrm{IP}_{3}$ concentrations for the three models of $\mathrm{IP}_{3}$ Rs. All our analytic results are checked against Gillespie-type simulations (Gillespie, 1977).

In all the plots solid curves correspond to the results obtained with the analytical approach, while isolated symbols denote Gillespie simulation points. Error bars on the latter denote standard error of the mean. In most cases, the error bars are smaller than the symbols. In all cases where the comparison was possible we find perfect agreement between the two methods. 


\subsection{9-state model}

In order to demonstrate that our analytic approach leads to the same results as other methods of calculation, we first reproduce the known results of ref. (Shuai et al., 2007) on opening times and probabilities in the 9-state model. Figures $5 \mathrm{a}$ and $5 \mathrm{~b}$ show the opening

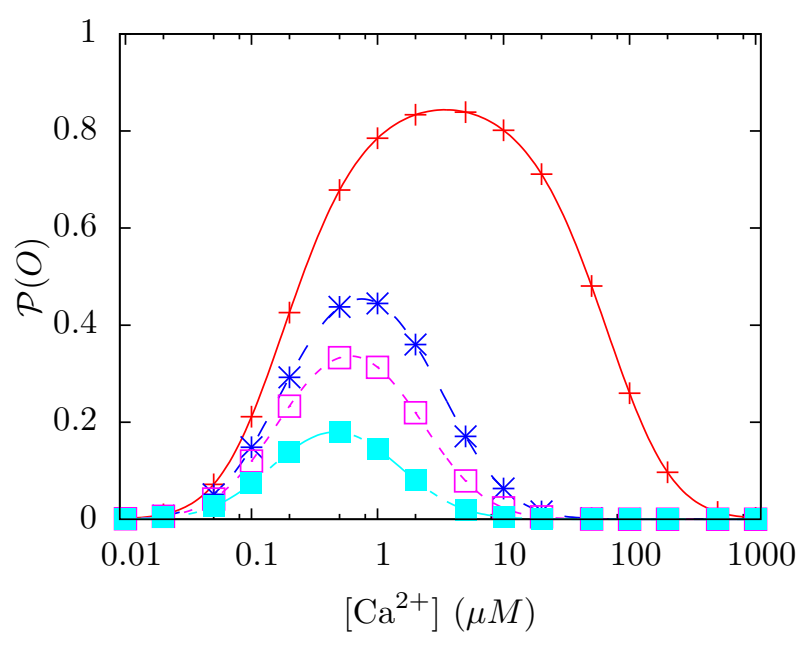

(a) Total opening probability (Eq. 22

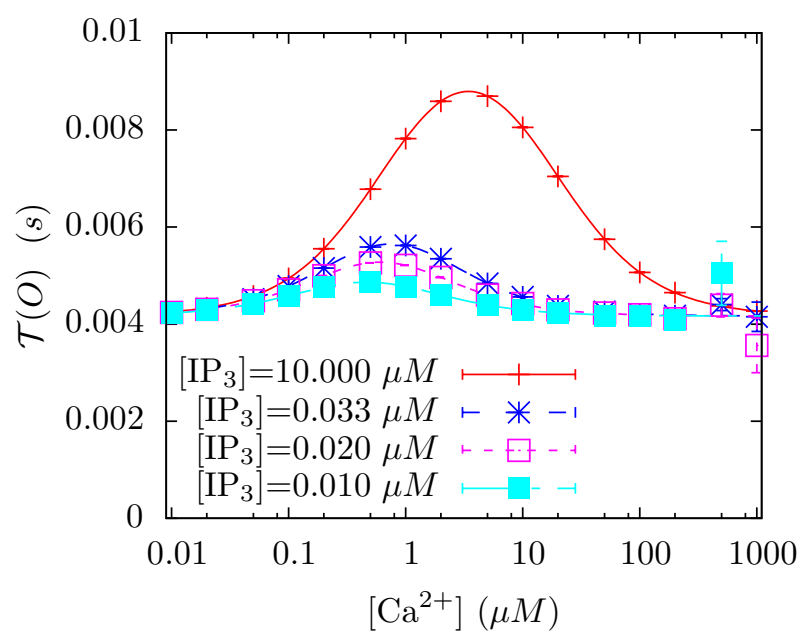

(b) Mean opening time (Eq. 23p

Figure 5: Total opening probability and mean opening time for the 9-state model for various $\mathrm{IP}_{3}$ concentrations as a function of $\mathrm{Ca}^{2+}$ concentration. The symbols show results from stochastic simulations and the solid lines the results obtained from the analytic expressions derived in the text. Error bars represent the standard error of the mean along the simulation history. Only errors on rare events, which occur at very high $\mathrm{Ca}^{2+}$ concentrations, are visibly large in the figures.

probability and the mean opening time (Eqs. 22 and 23), respectively. A different analytic derivation for these observables was provided in ref. Shuai et al. (2007).

Figures $6 \mathrm{a}$ and $6 \mathrm{~b}$ show observables associated to $L$-related opening events. Restricting to the case $N=4$ and $K_{t h}=3$, we show in particular the time spent in a 3- or 4-related opening event $\left(\mathcal{T}\left(A_{L}\right)\right.$, see Eq. 30 with $\left.L=3,4\right)$, and the probability that an opening event is 3- or 4-related (Eq. 32). For these quantities, analytic expressions have, to the best of our knowledge, not been established previously. Thus, these examples demonstrate the general applicability and usefulness of our approach. The advantage of analytic expressions of general validity is apparent: Expected times and their variances can be computed directly and exactly without the need for stochastic simulations. This is in particular valuable for rare events where computing times for stochastic simulations can dramatically increase.

\subsection{Comparing different models}

A particular strength of theoretical approaches to biology is the principle ability to suggest experiments which allow discriminating between conflicting models. We apply our theory to calculate measurable quantities which display a qualitatively different dependency on the external $\mathrm{Ca}^{2+}$ and $\mathrm{IP}_{3}$ concentrations and are therefore in principle suitable to differentiate 


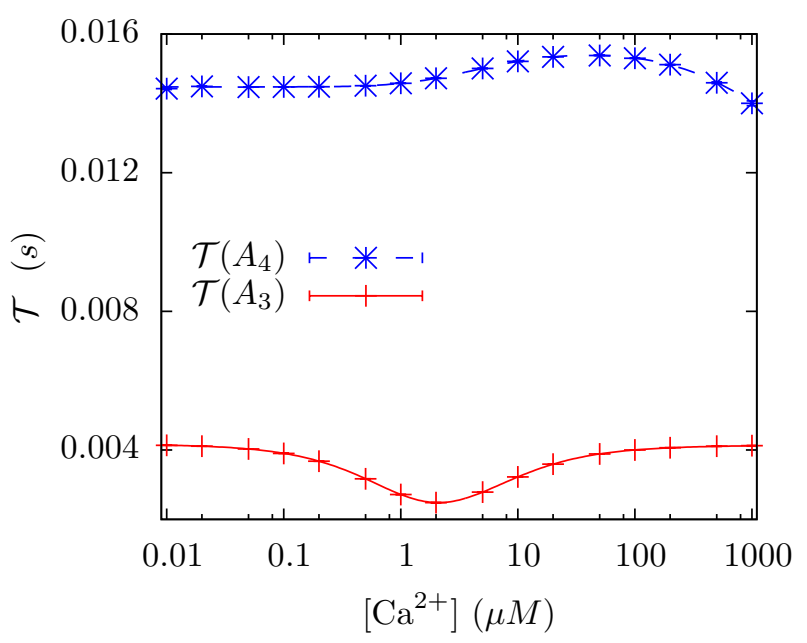

(a) Time spent in $L$-related opening (Eq. 30

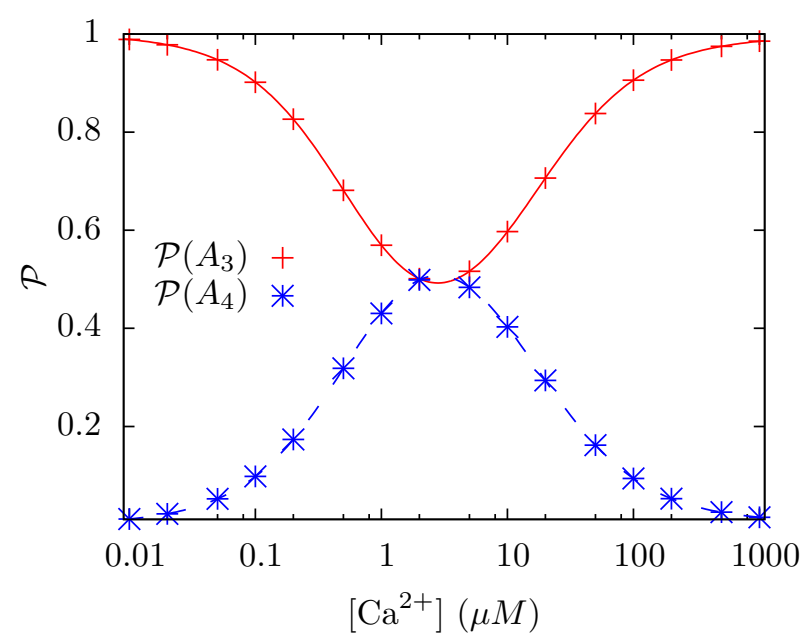

(b) Relative number of opening events (Eq. 32 )

Figure 6: Statistics for 3- and 4-related opening events for a concentration of $\left[\mathrm{IP}_{3}\right]=10 \mu M$. Shown are (a) the mean time spent in 3- and 4-related opening and (b) the relative frequencies of these events, determined by stochastic simulations (crosses) and through the analytic formulae (solid lines), as a function of $\mathrm{Ca}^{2+}$ concentration.

between the three discussed $\mathrm{IP}_{3} \mathrm{R}$ models. For the 9-state model we use the parameters as published in Shuai et al. (2007). For the 8-state model we simultaneously fit (see Supplementary Material S2) the total opening probability $\mathcal{P}(O)$ and the opening time $\mathcal{T}(O)$ to values obtained from the 9 -state model for various $\mathrm{Ca}^{2+}$ concentrations at $\left[\mathrm{IP}_{3}\right]=10 \mu M$ and $\left[\mathrm{IP}_{3}\right]=0.33 \mu M$ (the parameters do not strictly correspond to the best obtained fit but rather represent a trade off between fitness and reasonable magnitude; for details see Supplementary Material S2). The parameters of the global activation model are the same as the parameters of the 9-state model, with $a_{\mathcal{O}}=a_{0}$ and $b_{\mathcal{O}}=b_{0}$. All parameters are listed in Table 1 .

Experimentally accessible quantities are the opening and closing times of channels as well as the inter-spike intervals. In Fig. 7, the opening and closing times predicted by the three discussed models are depicted as a function of the $\mathrm{Ca}^{2+}$ concentration for a fixed value of $\left[\mathrm{IP}_{3}\right]$. Whereas the closing times show qualitatively similar behavior, the opening time predicted by the global activation model is clearly distinguishable from the 8- and 9-state models. In particular, $\mathcal{T}(O)$ is constant as a function of the $\mathrm{Ca}^{2+}$ concentration for the global activation model, which can be understood because the deactivation rate of the open state, $b_{\mathcal{O}}$, is a concentration independent constant.

Thus, we can conclude that the assumption of global channel activation is invalid since the predicted constant open time $\mathcal{T}(O)$ contradicts experimental measurements which display a bell-shaped dependency on the cytosolic $\mathrm{Ca}^{2+}$ concentration (Bezprozvanny et al., 1991). This calculation nicely illustrates the power of our approach since it allows to generate functional relations between measurable quantities and possible hidden mechanisms that can be studied in a parameter dependent manner.

While opening and closing times alone do not allow to test whether the 8- or the 9-state 


\begin{tabular}{cccc}
\hline & 9-state & 8-state & global \\
\hline$a_{1}$ & 60.0 & 56.9338 & 60.0 \\
$b_{1}$ & 0.216 & 0.200904 & 0.216 \\
$a_{2}$ & 0.2 & 0.190167 & 0.2 \\
$b_{2}$ & 3.2 & 13.591 & 3.2 \\
$a_{3}$ & 5.0 & 5.0 & 5.0 \\
$b_{3}$ & 4.0 & 3.35775 & 4.0 \\
$a_{4}$ & 0.5 & 0.5 & 0.5 \\
$b_{4}$ & 0.036 & 0.18777 & 0.036 \\
$a_{5}$ & 150.0 & 476.698 & 150.0 \\
$b_{5}$ & 120.0 & 88.3325 & 120.0 \\
$a_{0}$ & 540.0 & & \\
$b_{0}$ & 80.0 & & \\
$a_{\mathcal{O}}$ & & & 540.0 \\
$b_{\mathcal{O}}$ & & & 80.0 \\
\hline
\end{tabular}

Table 1: Model parameters used for Gillespie simulation and analytic calculations.

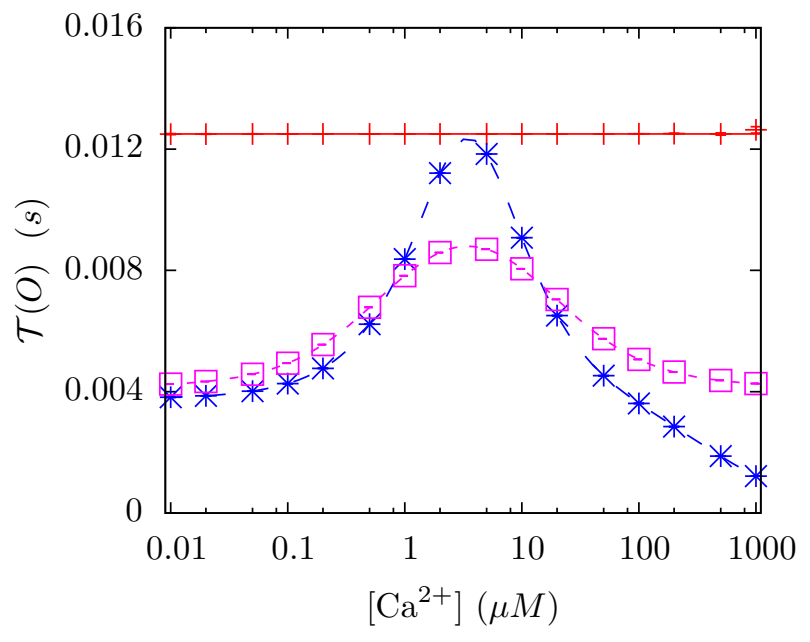

(a) Mean opening time (Eq. 23p

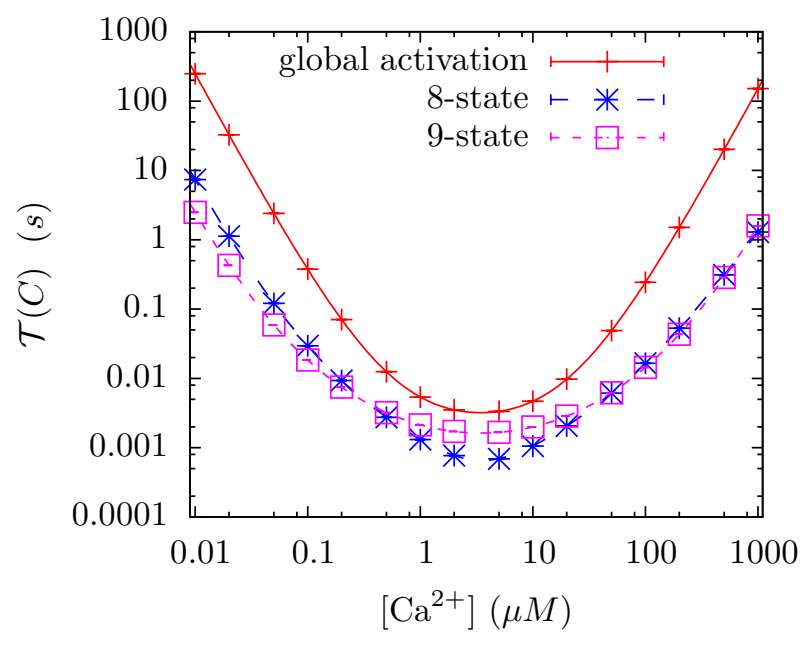

(b) Mean closing time (Eq. 24)

Figure 7: Comparison of mean opening and mean closing times in the 8-state, 9-state and global activation model for $\left[\mathrm{IP}_{3}\right]=10 \mu M$.

model describes experimental data better, this is possible by investigating the coefficient of variation $(\mathrm{CV})$ of the inter-spike intervals between opening events. Figure 8 shows the functional forms of the $\mathrm{CV}$ of the inter-spike intervals as functions of the $\mathrm{Ca}^{2+}$ and $\mathrm{IP}_{3}$ concentrations. All models show a qualitatively similar shape, with large changes of the $\mathrm{CV}$ being observed for $\mathrm{Ca}^{2+}$ concentrations between $\left[\mathrm{Ca}^{2+}\right]=0.1 \mu M$ and $\left[\mathrm{Ca}^{2+}\right]=10 \mu M$, and the largest changes for low $\mathrm{IP}_{3}$ concentrations with a local maximum around $\left[\mathrm{Ca}^{2+}\right]=2 \mu M$. 


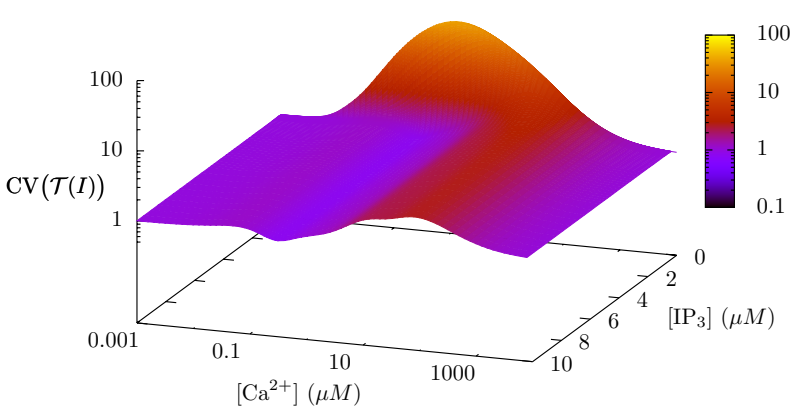

(a) 8-state model

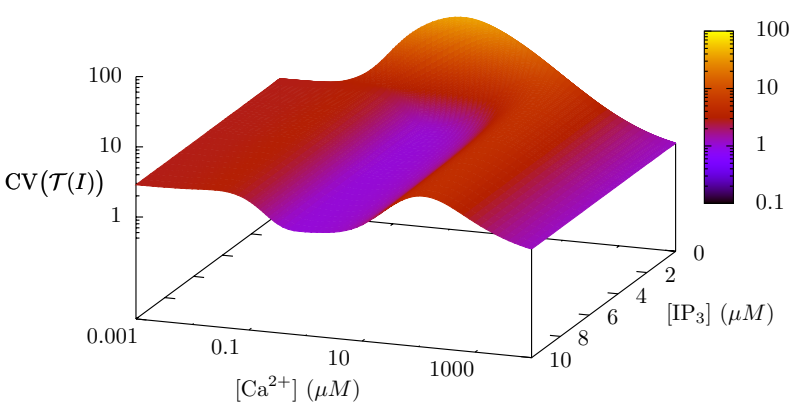

(b) 9-state model

Figure 8: Coefficient of variation of inter-spike intervals (Eq. 37) as function of $\mathrm{IP}_{3}$ and $\mathrm{Ca}^{2+}$ concentration.

Only for the case of the 8-state model the base-level of the CV at low concentrations reaches a value around 1, meaning that the inter-spike interval distributions collapse to an exponentiallike functional form.

This feature may be used to design experiments specifically targeting low $\mathrm{Ca}^{2+}$ concentrations to distinguish among different models.

\subsection{Receptor channels with an arbitrary number of subunits}

For the $\mathrm{IP}_{3} \mathrm{R}$ it is established that the internal structure consists of four subunits (Suhara et al., 2006; Mikoshiba, 1993; Michikawa et al., 1994; Jiang et al., 2002). However, in nature complexes with an almost arbitrary number of subunits exist (Unwin, 1989). With the theoretical analysis presented here, it is in principle possible to distinguish between different models describing the internal structure of a receptor channel. We consider a generalized 9-state model by assuming that a channel consisting of $N$ subunits is open if at least $K_{t h}$ subunits are in an active state. We illustrate the effect of a different internal receptor structure on the dynamic channel properties for the two cases of a 5-subunit channel which opens with at least 3 active subunits $\left(N=5, K_{t h}=3\right)$ and a 7 -subunit channel which also opens if at least 3 of the subunits are in the activated state $\left(N=7, K_{t h}=3\right)$.

The functional form of the CV of the inter-spike intervals are plotted in Fig. 9 as functions of the $\mathrm{Ca}^{2+}$ and $\mathrm{IP}_{3}$ concentrations for the two hypothetical channel configurations. The shape of the functions display peculiar features. In particular in the regime of high $\mathrm{IP}_{3}$ concentrations the $\mathrm{CV}$ exhibits an interesting multimodal form for $N=7$. This demonstrates the capability of our analytic approach to distinguish between similar models which only differ in the internal configuration of the channel.

\section{Conclusions}

The emergence of higher (mesoscopic) levels of organization from a lower level dynamics is a characteristic feature of complex systems. An established technique to study mesoscopic properties resulting from underlying stochastic processes is by detailed numerical simulations (Gillespie, 1977). Alternatively, analytical approaches have been developed to determine how the lower-level processes can lead to observable mesoscopic behaviors such as bursts 


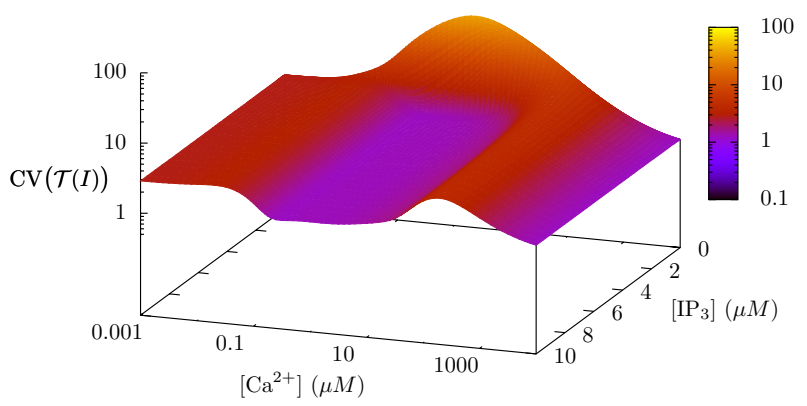

(a) $N=5, K_{t h}=3$

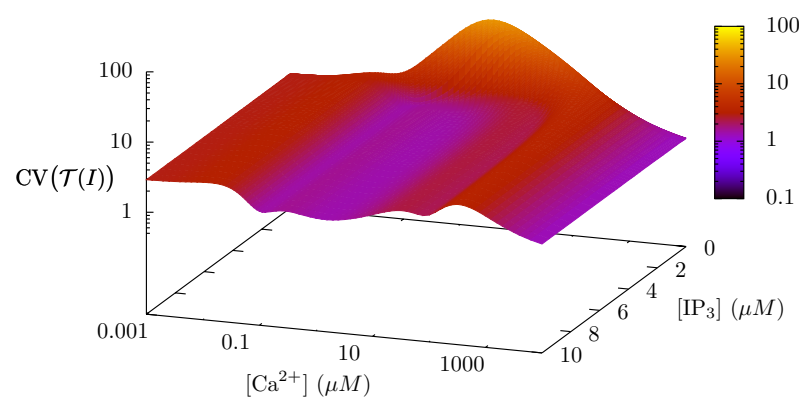

(b) $N=7, K_{t h}=3$

Figure 9: Coefficient of variation of inter-spike intervals (Eq. 37) as function of $\mathrm{IP}_{3}$ and $\mathrm{Ca}^{2+}$ concentration for models with different numbers of subunits. Both models assume that a channel opens if at least $K_{t h}=3$ subunits are in the activated state. The results in the left panel are computed for a hypothetical channel with 5 subunits, the right panel for 7 subunits.

of ion channels (Colquhoun and Hawkes, 1982). These analytical approaches allow in principle for an exact definition and calculation of transition probability densities and thus for a fully detailed description of the system properties, as they are based on the spectral expansion of the transition matrix. Therefore they rely on solving the corresponding eigenvalue problem and require following the whole time-evolution of the system. In this work we have developed an alternative analytic approach allowing for a direct computation of statistical properties of mesoscopic quantities from the underlying Markovian dynamics. Our methodology provides an attractive computational improvement able to circumvent time-consuming stochastic simulations and also to capture rare events in their entirety. In contrast to other analytical approaches (Colquhoun and Hawkes, 1982), our method is not able to determine the full probability density. However, due to the use of efficient recursive formulae, applicable to even very large systems, the present approach is able to provide analytic equations for the computation of the experimental predominant first statistical moments at the mesoscopic level from elementary microscopic rate constants.

To illustrate the potential of our general theoretical developments, we have studied the dynamics of three stochastic models of the so-called $\mathrm{IP}_{3}$ receptor channels, i.e. calcium channels releasing calcium from intracellular stores in a calcium and $\mathrm{IP}_{3}$ dependent manner. These are of high interest since their local properties shape cell-wide $\mathrm{Ca}^{2+}$ signals (Rüdiger et al., 2007; Skupin et al., 2010; Rüdiger et al., 2012) and are thus responsible for a variety of physiological processes (Berridge et al., 1998). In this context, our approach can be seen as a generalized and applicable method to predict mesoscopic behavior from underlying complex microscopic dynamics, which was for instance explicitly approximated for clustered $\mathrm{IP}_{3}$ receptors (Higgins et al., 2009). A slightly different approach for characterizing the dynamics of such a mesoscopic system was recently developed that is based on waiting time distributions of observable mesoscopic variables (Moenke et al., 2012). Due to the non-Markovian character of the mesoscopic dynamics, the considered waiting-time probabilities are non-exponential making calculations of the statistical moments of the dynamics based on Laplace transformation potentially problematic. Using reasonable approximations for the channel waiting-time 
distributions, the approach, however, enables direct and fast simulations of $\mathrm{Ca}^{2+}$ dynamics that fits experimental results nicely even though it does not make reference to a mechanistic molecular channel model.

We demonstrate the general applicability of our theory by deriving analytic formulae for mesoscopic quantities which previously were only accessible through stochastic simulations. These quite complex mesoscopic quantities, corresponding to channel opening events in which only a certain number of subunits are activated, are illustrative for understanding how the average channel opening times and probabilities are governed by the molecular structure of the receptors.

As a key application of our theoretical concepts we systematically investigate alternative models of the $\mathrm{IP}_{3}$ receptor channels and predict experimentally accessible mesoscopic quantities which can be used to discriminate between the models and establish which mechanistic model best describes the experimental observations. In particular, we have shown that the assumption of the global activation mechanism contradicts experimental data because it predicts a $\mathrm{Ca}^{2+}$ independent open time whereas a bell-shaped dependency is observed in experiments. Such ability to distinguish between competing mechanistic models is a key motivation for theoretical approaches to biology in general. A particular strength of analytic formulae is that they automatically capture rare events, which in stochastic simulations may easily be missed or are only accurately described with extremely long simulations. However, precisely these rare events are at the basis of the intermittency properties (Frisch, 1996) of complex dynamic systems and neglecting rare events may result in an inaccurate description of the dynamic properties of the investigated system.

While the investigated models all represent closed systems without external fluxes into or out of the system, our approach is applicable also to open systems in stationary state. The only assumption that enters our theoretical approach is that of stationarity, which means that the sum of the probability rates leading to a microstate should equal the sum of the probability rates of processes leaving that state, see Eq.2. For the calculation of all quantities which do not depend on stationary probabilities, including all those which are conditional on the residence of the system in a particular microstate, no modification is necessary for open systems. Those quantities depending on the stationary probability distribution also keep their functional form, but the presence of external fluxes requires a modification of the calculation of the stationary probabilities (Eq. 3). Let $v_{n}^{\text {in }}$ and $v_{n}^{\text {out }}$ denote the external fluxes into and out of microstate $n$, respectively. For the fluxes leaving the system, we can assign rate constants analogous to Eq. 1 and write

$$
v_{n}^{\text {out }}=p_{n} k_{n}^{\text {out }}
$$

leading to the balance equation

$$
v_{n}^{\text {in }}+\sum_{m} p_{m} k_{m n}=p_{n}\left(k_{n}^{\text {out }}+\sum_{l} k_{n l}\right),
$$

from which the stationary, non-equilibrium, probabilities can be determined by solving the inhomogeneous linear system of equations

$$
\sum_{m} p_{m}\left(k_{m n}-\delta_{m n}\left(k_{m}^{\text {out }}+\sum_{\ell} k_{m \ell}\right)\right)=-v_{n}^{\text {in }} .
$$


The derivation of the expressions for second moments of the dwell time distributions was based on the assumption that the underlying Markovian microscopic process is a Poisson process. The same assumption is fundamental for the Gillespie algorithm (Gillespie, 1977) on which stochastic simulations are based. For atomic and molecular interactions, this is a reasonable assumption which also underlies the derivation of the mass-action kinetic rate law for chemical reactions. Under this condition, there is no principle obstacle to derive expressions for the higher moments of the dwell time distribution in mesostates. With such expressions available the distribution of residence times can be approximated by analytic expressions with in principle arbitrary accuracy. The limitations of the applicability of this approach will probably lie in an increasing complexity of the resulting expressions and a concomitant difficulty of their numerical evaluation. However, even only with the analytic expressions for first and second moments presented in this work, the possibility has been established to extract relevant information about mesoscopic and experimentally accessible quantities for model discrimination and refinement.

\section{Acknowledgments}

The authors would like to express their gratitude to Prof. Reinhart Heinrich, who was the focal point of our collaboration but sadly deceased far too early.

They also wish to thank Prof. Michael Pusch for an enlightening discussion.

NC, KJ, SM and GCR acknowledge the Galileo Galilei Institute for Theoretical Physics for the hospitality offered to them, in the occasion of the workshop "New Frontiers in Lattice Gauge Theories", while this work was brought to an end. GCR would like to thank MIUR (Italy) for partial support under the PRIN contract number 20093BMNPR. SM would like to thank MIUR (Italy) for partial support under the PRIN contract number 20083Y4Y7. OE and NC were supported by the Scottish Funding Council through the Scottish Universities Life Science Alliance (SULSA). NC and AS are supported by the knowledge transfer program by the health transfer initiative of the Luxembourg government.

\section{Author contributions}

All authors designed the study during scientific discussions. NC implemented the algorithms and performed all numerical simulations. OE had the original idea of historydependent mesostate characteristics. NC, OE and GCR performed the analytic calculations. AS provided support in code development, contributed to model development and supported analytic calculations. AS and SM were instrumental in guiding the project towards biological relevance. KJ provided expertise in the mathematical and statistical formalisms. All authors wrote the paper. 
Supplementary Material 


\section{S1.1. Detailed derivation of $P(U \rightarrow k \in V \mid i \in U \wedge U \rightarrow V)$}

In this section we derive in detail the formula of the matrix elements $M_{i j}$ (Eq. 9 in the main text), which describes the process of visiting another state $j \neq i$ within $U$ before leaving to $V$.

We first calculate the probability that the system leaves directly to any microstate of $V$, under the condition that from $i \in U$ the next mesostate is $V$, by summing over $k$ in Eq. 8 in the main text

$$
\frac{\sum_{k^{\prime} \in V} \pi_{i k^{\prime}}}{P(U \rightarrow V \mid i \in U)} .
$$

So, conversely, the conditional probability that the system first visits any other microstate in $U$, and later leaves to mesostate $V$, is

$$
1-\frac{\sum_{k^{\prime} \in V} \pi_{i k^{\prime}}}{P(U \rightarrow V \mid i \in U)} .
$$

Given that the next mesostate transition is $U \rightarrow V$, but first a microstate in $U$ is visited, the conditional probability that this microstate is $j \in U$ is

$$
\frac{P(i \in U \rightarrow j \in U \wedge U \rightarrow V)}{\sum_{i^{\prime} \in U} P\left(i \in U \rightarrow i^{\prime} \in U \wedge U \rightarrow V\right)}=\frac{\pi_{i j} P(U \rightarrow V \mid j \in U)}{\sum_{i^{\prime} \in U} \pi_{i i^{\prime}} P\left(U \rightarrow V \mid i^{\prime} \in U\right)} .
$$

Altogether this leads to

$$
M_{i j}=P(i \in U \rightarrow j \in U \mid U \rightarrow V)=\left(1-\frac{\sum_{k^{\prime} \in V} \pi_{i k^{\prime}}}{P(U \rightarrow V \mid i \in U)}\right) \frac{\pi_{i j} P(U \rightarrow V \mid j \in U)}{\sum_{i^{\prime} \in U} \pi_{i i^{\prime}} P\left(U \rightarrow V \mid i^{\prime} \in U\right)} .
$$

\section{S1.2. Derivation of more complex dwell times}

Time spent in mesostate $U$ when the system is in $i \in U$ and in the next mesostate transition it arrives in $k \in V$. This quantity differs from Eq. 17 in the main text by the requirement that the system arrives in a specific microstate $k \in V$, therefore the matrix elements needed for its derivation are different. Apart from that the derivation is similar and one obtains

$$
\begin{aligned}
& T(U \mid i \in U \wedge U \rightarrow k \in V)=\tau_{i} \\
& +\sum_{j \in U} \underbrace{P(i \in U \rightarrow j \in U \mid U \rightarrow k \in V)}_{N_{i j}} T(U \mid j \in U \wedge U \rightarrow k \in V) \\
& T(U \mid i \in U \wedge U \rightarrow k \in V)=\sum_{j \in U}(\mathbf{1}-\mathbf{N})_{i j}^{-1} \tau_{j},
\end{aligned}
$$

where the matrix elements $N_{i j}$ are

$$
P(i \in U \rightarrow j \in U \mid U \rightarrow k \in V)=\left[\left(1-\frac{\pi_{i k}}{P(U \rightarrow k \in V \mid i \in U)}\right) \frac{\pi_{i j} P(U \rightarrow k \in V \mid j \in U)}{\sum_{i^{\prime} \in U} \pi_{i i^{\prime}} P\left(U \rightarrow k \in V \mid i^{\prime} \in U\right)}\right]
$$


For the corresponding second raw moment one then gets under the assumption of exponentially distributed $\tau_{i}$

$$
T^{(2)}(U \mid i \in U \wedge U \rightarrow k \in V)=2 \sum_{j \in U}(\mathbf{1}-\mathbf{N})_{i j}^{-1} \tau_{j} T(U \mid j \in U \wedge U \rightarrow k \in V) .
$$

Time spent in mesostate $U$ given that the mesostate transition chain is $W \rightarrow U \rightarrow V$. The formulae derived in the main text allow us to calculate probabilities and dwell times of arbitrary complicated transition chains. For example, the time spent in mesostate $U$, given that the mesostate transition chain is $W \rightarrow U \rightarrow V$, is

$$
T(U \mid W \rightarrow U \rightarrow V)=\sum_{j \in U} P(W \rightarrow j \in U \mid W \rightarrow U \rightarrow V) T(U \mid j \in U \wedge U \rightarrow V),
$$

where

$$
P(W \rightarrow j \in U \mid W \rightarrow U \rightarrow V)=\frac{P(W \rightarrow j \in U \mid W \rightarrow U) P(U \rightarrow V \mid j \in U)}{\sum_{j^{\prime} \in U} P\left(W \rightarrow j^{\prime} \in U \mid W \rightarrow U\right) P\left(U \rightarrow V \mid j^{\prime} \in U\right)}
$$

is the probability that in the chain $W \rightarrow U \rightarrow V$ the first visited microstate in $U$ is $j$.

The corresponding second raw moment is calculated by weighting each $T^{(2)}(U \mid i \in U \wedge$ $U \rightarrow V)$ with its probability (Eq. S1.9)

$$
T^{(2)}(U \mid W \rightarrow U \rightarrow V)=\sum_{j \in U} P(W \rightarrow j \in U \mid W \rightarrow U \rightarrow V) T^{(2)}(U \mid j \in U \wedge U \rightarrow V) .
$$

Similar to Eq. 11 in the main text, $T(U \mid W \rightarrow U \rightarrow V)$ and $T^{(2)}(U \mid W \rightarrow U \rightarrow V)$ are conditional only on mesostates (not on a particular microstate) and therefore depend on the stationary probabilities.

Time spent in mesostate $U$ when system is in $i \in U$ given that the mesostate transition chain is $U \rightarrow V \rightarrow Z$. For a transition chain with more than one mesostate visited "in the future", the matrix that needs to be inverted changes, but the derivation follows the same procedure as used when deriving simpler dwell times. We will derive the dwell time in $U$ where the next mesostates will be $V$ and $Z$

$$
T(U \mid i \in U \wedge U \rightarrow V \rightarrow Z)=\tau_{i}+\sum_{j \in U} P(i \in U \rightarrow j \in U \mid U \rightarrow V \rightarrow Z) T(U \mid j \in U \wedge U \rightarrow V \rightarrow Z),
$$

leading to

$$
T(U \mid i \in U \wedge U \rightarrow V \rightarrow Z)=\sum_{j \in U}\left(\mathbf{1}-\mathbf{M}^{U V Z}\right)_{i j}^{-1} \tau_{j}
$$

where the matrix elements are $M_{i j}^{U V Z}=P(i \in U \rightarrow j \in U \mid U \rightarrow V \rightarrow Z)$. Similar to Eq. 9 in the main text, these can be calculated in the following way

$$
\begin{aligned}
P(i \in U & \rightarrow j \in U \mid U \rightarrow V \rightarrow Z) \\
& =\left(1-\frac{\sum_{k^{\prime} \in V} P\left(i \in U \rightarrow k^{\prime} \in V \wedge U \rightarrow V \rightarrow Z\right)}{P(U \rightarrow V \rightarrow Z \mid i \in U)}\right) \frac{P(i \in U \rightarrow j \in U \wedge U \rightarrow V \rightarrow Z)}{\sum_{j^{\prime} \in U} P\left(i \in U \rightarrow j^{\prime} \in U \wedge U \rightarrow V \rightarrow Z\right)} \\
& =\left(1-\frac{\sum_{k^{\prime} \in V} \pi_{i k^{\prime}} P\left(V \rightarrow Z \mid k^{\prime} \in V\right)}{P(U \rightarrow V \rightarrow Z \mid i \in U)}\right) \frac{\pi_{i j} P(U \rightarrow V \rightarrow Z \mid j \in U)}{\sum_{j^{\prime} \in U} \pi_{i j^{\prime}} P\left(U \rightarrow V \rightarrow Z \mid j^{\prime} \in U\right)},
\end{aligned}
$$


where the probability that from $i \in U$ the mesostates $V$ and $Z$ are visited is

$$
P(U \rightarrow V \rightarrow Z \mid i \in U)=\sum_{k^{\prime} \in V} P\left(U \rightarrow k^{\prime} \in V \mid i \in U\right) P\left(V \rightarrow Z \mid k^{\prime} \in V\right) .
$$

Time spent in mesostate $U$ given that the mesostate transition chain is $W \rightarrow U \rightarrow V \rightarrow Z$. Here we will derive the dwell time in $U$ when the previous mesostate was $W$ and the next will be $V$ and then $Z$. Similar to Eq. S1.8, we write this as

$$
T(U \mid W \rightarrow U \rightarrow V \rightarrow Z)=\sum_{j \in U} P(W \rightarrow j \in U \mid W \rightarrow U \rightarrow V \rightarrow Z) T(U \mid j \in U \wedge U \rightarrow V \rightarrow Z)
$$

where the first term corresponds to Eq. S1.9

$$
P(W \rightarrow j \in U \mid W \rightarrow U \rightarrow V \rightarrow Z)=\frac{P(W \rightarrow j \in U \mid W \rightarrow U) P(U \rightarrow V \rightarrow Z \mid j \in U)}{\sum_{j^{\prime} \in U} P\left(W \rightarrow j^{\prime} \in U \mid W \rightarrow U\right) P\left(U \rightarrow V \rightarrow Z \mid j^{\prime} \in U\right)},
$$

and the second term is the dwell time derived in the previous paragraph (Eq. $\mathrm{S} 1.12$ ).

The examples presented above illustrate how an arbitrary complex quantity can be expressed by quantities of lower complexity.

\section{S1.3. Derivation of more complex quantities for mesostate subsets}

For the calculations in Sect. 4 of the main text, we require the time spent in mesostate $U$ given that the mesostate transition chain is $W \rightarrow U \rightarrow V^{\prime}: V \rightarrow Z$, which is given by

$$
T\left(U \mid W \rightarrow U \rightarrow V^{\prime}: V \rightarrow Z\right)=\sum_{j \in U} P\left(W \rightarrow j \in U \mid W \rightarrow U \rightarrow V^{\prime}: V \rightarrow Z\right) T\left(U \mid j \in U \wedge U \rightarrow V^{\prime}: V \rightarrow Z\right) .
$$

The probability which enters Eq. S1.17 takes the form

$$
P\left(W \rightarrow j \in U \mid W \rightarrow U \rightarrow V^{\prime}: V \rightarrow Z\right)=\frac{P(W \rightarrow j \in U \mid W \rightarrow U) P\left(U \rightarrow V^{\prime}: V \rightarrow Z \mid j \in U\right)}{\sum_{j^{\prime} \in U} P\left(W \rightarrow j^{\prime} \in U \mid W \rightarrow U\right) P\left(U \rightarrow V^{\prime}: V \rightarrow Z \mid j^{\prime} \in U\right)}
$$

The matrix elements $M_{i j}^{U V^{\prime}: V Z}=P\left(i \in U \rightarrow j \in U \mid U \rightarrow V^{\prime}: V \rightarrow Z\right)$ needed for the derivation of $T\left(U \mid i \in U \wedge U \rightarrow V^{\prime}: V \rightarrow Z\right)$ can be calculated in analogy to Eq. S1.13. For the calculation, the transition chains $U \rightarrow V \rightarrow Z$ in the probabilities have to be replaced by the chains $U \rightarrow V^{\prime}: V \rightarrow Z$. These probabilities are defined in Eq. 12 in the main text. 


\section{S2.1. Fitting 8-state model parameters}

To determine parameters for the 8-state model $\left(p^{8 \text {-state }}\right)$, we have first calculated the total opening probability $\mathcal{P}(O)$ and the opening time $\mathcal{T}(O)$ of the 9-state model at various $\mathrm{Ca}^{2+}$ and $\mathrm{IP}_{3}$ concentrations using the parameters $p^{9 \text {-state }}$ from Shuai et al. (2007), and used these values as input for a fitting procedure.

As the two observables $\mathcal{P}(O)$ and $\mathcal{T}(O)$ do not depend on all parameters, the fitting procedure was reduced to the parameters $a_{1}, b_{1}, a_{2}, b_{2}, b_{3}, b_{4}, a_{5}, b_{5}$, with the parameters $a_{3}=5$ and $a_{4}=0.5$ held fixed.

To have equally logarithmically spaced calcium concentrations we took $\left[\mathrm{Ca}^{2+}\right]=10^{x} \mu M$ with $x=-0.7,-0.6, \ldots, 1.9,2.0$, leading to concentrations in the range $\left[\mathrm{Ca}^{2+}\right]=[0.2,100] \mu M$. For the $\mathrm{IP}_{3}$ concentrations we have chosen $\left[\mathrm{IP}_{3}\right]=10 \mu M$ and $\left[\mathrm{IP}_{3}\right]=0.33 \mu M$.

We used the genetic optimization package rgenoud (Mebane and Sekhon, 2011) to minimize a $\chi^{2}$ function where the $\mathcal{P}(O)$ and $\mathcal{T}(O)$ contributions are weighed with the coefficients $W_{\mathcal{P}(O)}$ and $W_{\mathcal{T}(O)}$

$$
\begin{aligned}
\chi^{2}= & W_{\mathcal{P}(O)} \sum_{\mathrm{Ca}^{2+}, \mathrm{IP}_{3}}\left(\mathcal{P}(O)\left(p^{9 \text {-state }}, \mathrm{Ca}^{2+}, \mathrm{IP}_{3}\right)-\mathcal{P}(O)\left(p^{8 \text {-state }}, \mathrm{Ca}^{2+}, \mathrm{IP}_{3}\right)\right)^{2} \\
& +W_{\mathcal{T}(O)} \frac{1}{s} \sum_{\mathrm{Ca}^{2+}, \mathrm{IP}_{3}}\left(\mathcal{T}(O)\left(p^{9 \text {-state }}, \mathrm{Ca}^{2+}, \mathrm{IP}_{3}\right)-\mathcal{T}(O)\left(p^{8 \text {-state }}, \mathrm{Ca}^{2+}, \mathrm{IP}_{3}\right)\right)^{2} .
\end{aligned}
$$

Note that $\frac{1}{s}$ is required to make the contribution of the opening times dimensionless.

We arbitrarily chose $W_{\mathcal{P}(O)}=W_{\mathcal{T}(O)}=0.5$. Depending on the given random seed, the fitted parameters differ dramatically while having compatible values of $\chi^{2}$, indicating that the fitness landscape is rather flat but bumpy. We therefore decided to restrain all parameters between 0.01 and 500 (using genoud's Domains parameter) to look for parameters of reasonable size, and randomly chose one of the solutions.

We should emphasize here that this result does not correspond to a proper fit, but rather represents a solution of $\chi^{2}$-minimization leading to reasonable values of the parameters. 


\section{References}

Allbritton, N., Meyer, T., Sryer, L., 1992. Range of messenger action of calcium ion and inositol 1,4,5 trisphosphate. Science 258, 1812-1815.

Ball, F.G., Milne, R.K., Yeo, G.F., 2000. Stochastic models for systems of interacting ion channels. Mathematical Medicine and Biology 17, 263-293. URL: http://imammb. oxfordjournals.org/content/17/3/263.abstract, doi:10.1093/imammb/17.3.263.

Berridge, M., 1997. Elementary and global aspects of calcium signalling. J.Physiol. 499, 291-306.

Berridge, M., Bootman, M., Lipp, P., 1998. Calcium - a life and death signal. Nature 395, 645-648.

Bezprozvanny, I., Ehrlich, B., 1994. Inositol(1,4,5)-trisphosphate ( $\left.\mathrm{InsP}_{3}\right)$-gated channels from cerebellum: conduction properties for divalent cations and regulation by intraluminal calcium. J.Gen.Physiol. 104, 821-856.

Bezprozvanny, I., Watras, J., Ehrlich, B., 1991. Bell-shaped calcium-response curves of Ins $(1,4,5) \mathrm{P}_{3^{-}}$and calcium-gated channels from endoplasmatic reticulum of cerebellum. Nature $351,751-754$.

Colquhoun, D., Hawkes, A.G., 1982. On the stochastic properties of bursts of single ion channel openings and of clusters of bursts. Philos Trans R Soc Lond B Biol Sci 300, 1-59.

De Young, G.W., Keizer, J., 1992. A single-pool inositol 1,4,5-trisphosphate-receptor-based model for agonist-stimulated oscillations in ca2+ concentration. Proceedings of the National Academy of Sciences 89, 9895-9899. URL: http://www.pnas.org/content/89/20/ 9895. abstract.

Dellis, O., Dedos, S.G., Tovey, S.C., Taufiq-Ur-Rahman, Dubel, S.J., Taylor, C.W., 2006. $\mathrm{Ca}^{2+}$ entry through plasma membrane $\mathrm{IP}_{3}$ receptors. Science 313, 229-233. URL: http: //dx.doi.org/10.1126/science.1125203, doi:10.1126/science.1125203

Falcke, M., Malchow, D. (Eds.), 2003. Understanding Calcium Dynamics - Experiments and Theory. Lecture Notes in Physics, Vol.623, Springer, Berlin Heidelberg New York.

Frisch, U., 1996. Turbulence: The legacy of A. N. Kolmogorov. Journal of Fluid Mechanics 317, 407-410. URL: http://dx.doi.org/10.1017/S0022112096210791, doi:10.1017/ S0022112096210791.

Gillespie, D.T., 1977. Exact stochastic simulation of coupled chemical reactions. J. Phys. Chem. 81, 2340-2361. doi:10.1021/j100540a008.

Gin, E., Falcke, M., Wagner, L.E., Yule, D.I., Sneyd, J., 2009. A kinetic model of the inositol trisphosphate receptor based on single-channel data. Biophys J 96, 4053-4062. URL: http: //dx.doi.org/10.1016/j.bpj.2008.12.3964, doi:10.1016/j.bpj.2008.12.3964. 
Heinrich, R., Rapoport, T.A., 2005. Generation of nonidentical compartments in vesicular transport systems. J Cell Biol 168, 271-280. URL: http://dx.doi.org/10.1083/jcb. 200409087, doi:10.1083/jcb.200409087.

Higgins, E.R., Schmidle, H., Falcke, M., 2009. Waiting time distributions for clusters of $\mathrm{IP}_{3}$ receptors. J Theor Biol 259, 338-349. URL: http://dx.doi.org/10.1016/j.jtbi.2009. 03.018, doi:10.1016/j.jtbi.2009.03.018.

Jiang, Q.X., Thrower, E., Chester, D., Ehrlich, B., Sigworth, F., 2002. Three-dimensional structure of the type 1 insositol 1,4,5-trisphosphate receptor at 24 a resolution. EMBO J. $21,3575-3581$.

La Penna, G., Morante, S., Perico, A., Rossi, G., 2004. Designing generalized statistical ensembles for numerical simulations of biopolymers. J Chem Phys 121, 10725-10741. URL: http://dx.doi.org/10.1063/1.1795694, doi:10.1063/1.1795694.

Laughlin, R.B., Pines, D., 2000. The theory of everything. Proc Natl Acad Sci U S A 97, $28-31$.

MacLennan, D., Rice, W., Green, N., 1997. The mechanism of Ca2+ transport by Sarco(Endo)plasmic Reticulum Ca2+-ATPases. J.Biol.Chem. 272, 28815-28818.

Mebane, Jr., W.R., Sekhon, J.S., 2011. Genetic optimization using derivatives: The rgenoud package for r. Journal of Statistical Software 42, 1-26. URL: http://www.jstatsoft. org/v42/i11.

Mézard, M., Parisi, G., Virasoro, M., 1987. Spin glass theory and beyond. World Scientific lecture notes in physics, World Scientific. URL: http://books.google.com/books?id= ZIF9QgAACAAJ.

Michikawa, T., Hamanaka, H., Otsu, H., Yamamoto, A., Miyawaki, A., Furuichi, T., Tashiro, Y., Mikoshiba, K., 1994. Transmembrane topology and sites of n-glycosylation of Inositol 1,4,5-trisphosphate receptor. J. Biol. Chem. 269, 9184-9189.

Mikoshiba, K., 1993. Inositol 1,4,5-trisphosphate receptor. Trends in Pharmacolical Sciences $14,86-89$.

Moenke, G., Falcke, M., Thurley, K., 2012. Hierarchic stochastic modelling applied to intracellular cajsup $2+i / \sup i$ signals. PLoS ONE 7, e51178. URL: http://dx.doi.org/10. 1371\%2F journal.pone.0051178, doi:10.1371/journal.pone.0051178.

Parisi, G., 1990. A simple model for the immune network. Proc Natl Acad Sci U S A 87, 429-433.

Rahman, T., Skupin, A., Falcke, M., Taylor, C., 2009. Clustering of $\mathrm{IP}_{3}$ receptors by $\mathrm{IP}_{3}$ retunes their regulation by $\mathrm{IP}_{3}$ and $\mathrm{Ca}^{2+}$. Nature 458, 655-659.

Rüdiger, S., Jung, P., Shuai, J.W., 2012. Termination of $\mathrm{Ca}^{2+}$ release for clustered $\mathrm{IP}_{3} \mathrm{R}$ channels. PLoS Comput Biol 8, e1002485. URL: http://dx.doi.org/10.1371/journal. pcbi.1002485, doi:10.1371/journal.pcbi.1002485. 
Rüdiger, S., Shuai, J.W., Huisinga, W., Nagaiah, C., Warnecke, G., Parker, I., Falcke, M., 2007. Hybrid stochastic and deterministic simulations of calcium blips. Biophys J 93, 1847-1857. URL:http://dx.doi.org/10.1529/biophysj.106.099879, doi:10.1529/ biophysj.106.099879.

Schrödinger, E., 1944. What is Life? Cambridge University Press.

Schwalger, T., Lindner, B., 2010. Theory for serial correlations of interevent intervals. The European Physical Journal - Special Topics 187, 211-221-221. URL: http://dx.doi.org/ 10.1140/epjst/e2010-01286-y, doi:10.1140/epjst/e2010-01286-y.

Shuai, J., Pearson, J.E., Foskett, J.K., Mak, D.O.D., Parker, I., 2007. A kinetic model of single and clustered IP3 receptors in the absence of $\mathrm{Ca} 2+$ feedback. Biophys J 93, 1151-1162. URL: http://dx.doi.org/10.1529/biophysj.107.108795, doi:10.1529/ biophysj.107.108795

Skupin, A., Falcke, M., 2009. From puffs to global $\mathrm{Ca}^{2+}$ signals: how molecular properties shape global signals. Chaos 19, 037111. URL: http://dx.doi.org/10.1063/1.3184537, doi:10.1063/1.3184537.

Skupin, A., Kettenmann, H., Falcke, M., 2010. Calcium signals driven by single channel noise. PLoS Comput Biol 6, e1000870. URL: http://dx.doi.org/10.1371/journal. pcbi.1000870, doi:10.1371/journal.pcbi.1000870.

Skupin, A., Kettenmann, H., Winkler, U., Wartenberg, M., Sauer, H., Tovey, S., Taylor, C., Falcke, M., 2008. How does intracellular $\mathrm{Ca}^{2+}$ oscillate: By chance or by the clock? Biophys. J. 94, 2404-2411.

Suhara, W., Kobayashi, M., Sagara, H., Hamadad, K., Goto, T., Fujimoto, I., Torimitsu, K., Mikoshiba, K., 2006. Visualization of inositol 1,4,5-trisphosphate receptor by atomic force microscopy. Neuroscience Letters 391, 102-107.

Unwin, N., 1989. The structure of ion channels in membranes of excitable cells. Neuron 3, 665-676. 